\title{
SERRE WEIGHTS FOR THREE-DIMENSIONAL ORDINARY GALOIS REPRESENTATIONS
}

\author{
STEFANO MORRA AND CHOL PARK
}

\begin{abstract}
Let $F / \mathbf{Q}$ be a CM field where $p$ splits completely and let $\bar{r}: \operatorname{Gal}(\overline{\mathbf{Q}} / F) \rightarrow$ $\mathrm{GL}_{3}\left(\overline{\mathbf{F}}_{p}\right)$ be a Galois representation whose restriction to $\mathrm{Gal}\left(\overline{\mathbf{Q}}_{p} / F_{w}\right)$ is ordinary and strongly generic for all places $w$ above $p$. In this paper we specify the set of Serre weights in which $\bar{r}$ can be modular. To this aim, we develop a technique in integral $p$-adic Hodge theory to describe extensions of rank-one Breuil modules.
\end{abstract}

\section{Contents}

1. Introduction

2. Integral $p$-adic Hodge theory, I: Preliminaries

3. Integral $p$-adic Hodge theory, II: Complements

4. Elimination of Galois types

4.1. The local Galois side

4.2. Galois types in niveau 1

4.3. Galois types in niveau 2

4.4. Galois types in niveau 3

5. Local/global compatibility and Serre weights

5.1. The space of automorphic forms on certain unitary groups 18

5.2. Potentially crystalline lifts and Serre weights 19

5.3. Modular weights and main results 23

5.4. Proof of Theorem 5.9 25

$\begin{array}{lr}\text { Acknowledgements } & 30\end{array}$

$\begin{array}{ll}\text { References } & 31\end{array}$

\section{INTRODUCTION}

Generalizations of the weight part in Serre's modularity conjecture ([Ser87]) have received considerable attention in recent years. Since the seminal work of Buzzard, Diamond, and Jarvis [BDJ10], substantial progress has been made and we now have a detailed description of such phenomena both for groups in higher semisimple rank ([Her09], [EGH13], [BLGG], [GG12]) or defined over extensions of $\mathbf{Q}$ ([Gee11], [GK], [GLS14], see also [GLS]).

In order to examine the situation in more detail, let us consider a CM field $F / \mathbf{Q}, F^{+}$ being its maximal totally real subfield and we let $\bar{r}: G_{F} \stackrel{\text { def }}{=} \operatorname{Gal}(\overline{\mathbf{Q}} / F) \rightarrow \mathrm{GL}_{n}\left(\overline{\mathbf{F}}_{p}\right)$ be a continuous Galois representation. Assume that $\bar{r}$ is modular: in our context, this means that there exists a totally definite unitary group $G$ defined over $F^{+}$such that the system of Hecke eigenvalues $\alpha_{\bar{r}}$ obtained from the semisimple conjugacy classes associated to the 
Frobenius elements (on the unramified locus of $\bar{r}$ ) appears in the 0-th cohomology of the space of mod- $p$ algebraic automorphic forms of some tame level:

$$
S^{G}\left(K^{p}, \overline{\mathbf{F}}_{p}\right) \stackrel{\text { def }}{=}\left\{f: G\left(F^{+}\right) \backslash G\left(\mathbf{A}_{F^{+}}^{\infty}\right) / K^{p} \rightarrow \overline{\mathbf{F}}_{p} \mid f \text { is locally constant }\right\}
$$

for some compact open level $K^{p} \leq G\left(\mathbf{A}_{F^{+}}^{\infty, p}\right)$ away from $p$.

Most of the efforts in the ongoing research are focused on the set $W_{\bmod }(\bar{r})$ of weights in which such $\bar{r}$ is modular, i.e., provided that $G$ admits an integral model $\mathcal{G}$ over $\mathcal{O}_{F^{+}, p} \stackrel{\text { def }}{=}$ $\mathcal{O}_{F^{+}} \otimes \mathbf{z} \mathbf{Z}_{p}$, the set $W_{\text {mod }}(\bar{r})$ of the irreducible smooth representations $V$ of $\mathcal{G}\left(\mathcal{O}_{F^{+}, p}\right)$ such that

$$
\operatorname{Hom}_{\mathcal{G}\left(\mathcal{O}_{F^{+}, p}\right)}\left(V, S^{G}\left(K^{p}, \overline{\mathbf{F}}_{p}\right)\left[\operatorname{ker} \alpha_{\bar{r}}\right]\right) \neq 0 .
$$

In particular, the weight part in Serre's conjectures gives a first description of the smooth $G\left(F_{p}^{+}\right)$-representation $S^{G}\left(K^{p}, \overline{\mathbf{F}}_{p}\right)\left[\operatorname{ker} \alpha_{\bar{r}}\right]$, an object whose nature is extremely badly understood and, according to the $p$-adic Langlands conjectures, shall depend only on $\left\{\left.\bar{r}\right|_{G_{F_{w}}}\right\}_{w \mid p}$.

Assume that $p$ splits completely in $F$ and that $\mathcal{G}\left(\mathcal{O}_{F_{w}}\right) \cong \mathrm{GL}_{n}\left(\mathcal{O}_{F_{w}}\right)$ for all $w \mid p$. When $n>2$, important insight towards the weight part in Serre's conjectures appears in [ADP02] and [Her09]. In particular, when the local parameters $\left.\bar{r}\right|_{G_{F_{w}}}$ are all tame, Herzig provides a set of weights $W^{?}(\bar{r})$, depending only on $\left\{\left.\bar{r}\right|_{G_{F_{w}}}\right\}_{w \mid p}$, for which $\bar{r}$ is modular, and which is obtained from level zero automorphic types naturally associated to the local datum $\left\{\left.\bar{r}\right|_{G_{F_{w}}}\right\}_{w \mid p}$. Progress towards [Her09], Conjecture 6.9 has recently been made when $\left.\bar{r}\right|_{G_{F_{w}}}$ is semisimple, cf. [EGH13], [BLGG] (see also [GG12]).

When $\left.\bar{r}\right|_{G_{F_{w}}}$ is not semisimple, much less is known. In [Gee11], Gee suggests a set of modular weights for $\bar{r}$, without requiring $\left.\bar{r}\right|_{G_{F_{w}}}$ to be tame but avoiding any reference to automorphic types, the set $W^{?}(\bar{r})$ being now described by means of crystalline lifts of $\left.\bar{r}\right|_{G_{F_{w}}}$. Evidence towards Gee's approach has first appeared in [GG12], where the authors prove that the weights defined by a non-vanishing condition on the ordinary part of the Hecke eigenspace $S^{G}\left(K^{p}, \overline{\mathbf{F}}_{p}\right)\left[\operatorname{ker} \alpha_{\bar{r}}\right]$ are described by ordinary crystalline lifts of $\left.\bar{r}\right|_{G_{F_{w}}}$.

The aim of this paper is to obtain evidence towards Serre-type conjectures for ordinary Galois representations in semisimple rank 2, developing the techniques introduced in [HM] where the maximally non-split case is considered. The submodule structure of $\left.\bar{r}\right|_{G_{F_{w}}}$ (for $w \mid p$ ) plays now a crucial role (as in $[\mathrm{BH}]$ ) and we define, for all possible configurations of the submodule structure in $\left.\bar{r}\right|_{G_{F_{w}}}$, a set of weights $W^{?}(\bar{r})$ in which $\bar{r}$ shall be modular. We remark that the set $W^{?}(\bar{r})$ is compatible with Herzig and Gee's conjectures ([Her09], [Gee11]) and with Gee-Geraghty's results on ordinary modularity -i.e. the ordinary weights appearing in $W^{?}(\bar{r})$ coincide with the set of ordinary weights determined by Gee and Geraghty in [GG12].

The main result of this paper is the following:

Theorem 1.1. Let $\bar{r}: G_{F} \rightarrow \mathrm{GL}_{3}\left(\overline{\mathbf{F}}_{p}\right)$ be a modular Galois representation. We assume that $p$ splits completely in $F$ and that for all places $w \mid p$ the Galois representation $\left.\bar{r}\right|_{G_{F}}$ is ordinary, strongly generic (cf. Definition 4.1), and of Loewy length less than 3.

Then one has

$$
W_{\text {mod }}(\bar{r}) \subseteq W^{?}(\bar{r}) .
$$

The case that $\left.\bar{r}\right|_{G_{F_{w}}}$ is maximally non-split (i.e., the Loewy length of $\left.\bar{r}\right|_{G_{F_{w}}}$ is 3) is treated in $[\mathrm{HM}]$.

Theorem 1.1 is a weight elimination result and is performed by integral $p$-adic Hodge theory and classical local/global compatibility. It relies on a crucial ingredient in integral $p$-adic Hodge theory, namely a splitting lemma (Lemma 3.2) which lets us determine the 
vanishing of a class $[c] \in H^{1}\left(G_{\mathbf{Q}_{p}}, \omega^{n}\right)$ ( $\omega$ denoting the mod $p$-cyclotomic character) by the only information coming from filtration on a Breuil module with descent data $\mathcal{M}$ such that $\left[\mathrm{T}_{\mathrm{st}}(\mathcal{M})\right]=[c]$.

We remark that Theorem 1.1 is consistent with the results of $\mathrm{D}$. Le on the moduli space of Fontaine-Laffaille modules [Le]. In forthcoming work, we are planning to generalize the previous result to arbitrary dimension and obtain partial results on the modularity of weights in $W^{?}(\bar{r})$.

The upper bound on the modular weights for $\bar{r}$ in Theorem 1.1 is expected to be the best possible result one can obtain via the classical weight elimination techniques. Understanding which weights in $W^{?}(\bar{\rho})$ are actually modular for $\bar{r}$ is on the other hand a very delicate problem. Following [BLGG] (building on the forthcoming [EGHS]), one can define a subset of obvious weights $W^{\text {? }, o b v}(\bar{\rho})$ by considering ordinary crystalline lifts of $\bar{\rho}$. One always has $W^{?, o b v}(\bar{\rho}) \subseteq W^{?}(\bar{\rho})$ and by combining Theorem 1.1 and [BLGG] Theorem 4.1.9, one obtains the following result:

Theorem 1.2. Let $F$ be an imaginary $C M$ field with maximal totally real subfield $F^{+}$ and suppose that $F / F^{+}$is unramified at all finite places, and that $p$ splits completely in $F$. Suppose that $\bar{r}: G_{F} \rightarrow \mathrm{GL}_{3}(\mathbf{F})$ is an irreducible representation with split ramification. Assume that there is a RACSDC automorphic representation $\Pi$ of $\mathrm{GL}_{3}\left(\mathbf{A}_{F}\right)$ of weight $\mu \in\left(\mathbf{Z}_{+}^{3}\right)_{0}^{\operatorname{Hom}(F, \mathbf{C})}$ and level prime to $p$ such that

$\circ \bar{r} \simeq \bar{r}_{p, i}(\Pi)$;

- For each place $w \mid p$ of $F,\left.r_{p, i}(\Pi)\right|_{G_{F_{w}}}$ is potentially diagonalizable;

$\circ \bar{r}\left(G_{F\left(\zeta_{p}\right)}\right)$ is adequate.

Assume further that for all places $w \mid p$ the Galois representation $\left.\bar{r}\right|_{G_{F_{w}}}$ are ordinary, strongly generic, and of Loewy length less than 3.

Then one has

$$
W^{?, o b v}(\bar{r}) \subseteq W_{\text {mod }}(\bar{r}) .
$$

The paper is organized as follows. In the remainder of the introduction we fix the notation that will be used throughout the paper. In Section 2, we quickly review integral $p$-adic Hodge theory, such as strongly divisible modules, Breuil-Kisin modules, Fontaine-Laffaille modules, and so on. In Section 3, we establish the necessary results from $p$-adic Hodge theory; for instance, we give enough conditions (Lemma 3.2) to determine if a rank-two Breuil module is split, which is the key lemma to perform the weight elimination. In Section 4, we eliminate the Galois types of rank-three Breuil modules that correspond to the local representations we impose, by the results we developed in Section 3. In Section 5, we first review the definitions of modular representations and modular weights, and then state and prove the main results, Theorems 5.9 and 5.10 .

Notation. Let $\overline{\mathbf{Q}}$ be an algebraic closure of $\mathbf{Q}$. All number field $F / \mathbf{Q}$ will be considered as subfield in $\overline{\mathbf{Q}}$ and we will write $G_{F} \stackrel{\text { def }}{=} \mathrm{Gal}(\overline{\mathbf{Q}} / F)$ to denote the absolute Galois group of $F$. For any rational prime $\ell \in \mathbf{Q}$, we fix algebraic closures $\overline{\mathbf{Q}}_{\ell}$ of $\mathbf{Q}_{\ell}$ and embeddings $\overline{\mathbf{Q}} \hookrightarrow \overline{\mathbf{Q}}_{\ell}$ which are compatible with the inclusions $G_{\mathbf{Q}_{\ell}} \hookrightarrow G_{\mathbf{Q}}$. In a similar fashion, we fix algebraic closures $\overline{\mathbf{F}}_{\ell}$ for the residue field $\mathbf{F}_{\ell}$ of $\mathbf{Q}_{\ell}$, compatibly with the natural morphisms at the level of the Galois groups. As above, all extensions of $\mathbf{Q}_{\ell}, \mathbf{F}_{\ell}$ will be considered as subfields in the fixed algebraic closure $\overline{\mathbf{Q}}_{\ell}, \overline{\mathbf{F}}_{\ell}$.

Let $f \geq 1$. We let $K_{0} \stackrel{\text { def }}{=} \mathbf{Q}_{p}$ be the unramified extension of $\mathbf{Q}_{p}$ of degree $f$, and write $K / K_{0}$ for the tamely totally ramified extension of $K_{0}$ of degree $e \stackrel{\text { def }}{=} p^{f}-1$. We consider the 
Eisenstein polynomial $E(u) \stackrel{\text { def }}{=} u^{e}+p$, and fix a root $\pi_{K}=\sqrt[e]{-p}$, which will be our choice for an uniformizer for $K / \mathbf{Q}_{p}$. If no confusion is possible, we will simply write $\pi$ instead of $\pi_{K}$. We finally let $k=\mathbf{F}_{p^{f}}$ be the residue field of $K$.

Let $E$ be a finite extension of $\mathbf{Q}_{p}$. We write $\mathcal{O}_{E}$ for its ring of integers, $\mathbf{F}$ for its residue field and $\varpi \in \mathcal{O}_{E}$ to denote an uniformizer. All the representations considered in this paper will be realized on vector spaces over $E$ or $\mathbf{F}$, which will be the fields of coefficients. We always assume that $E$ is large enough, i.e. that the Galois closure of $K$ over $\mathbf{Q}_{p}$ is contained in $E$ so that $\operatorname{Hom}\left(K, \overline{\mathbf{Q}}_{p}\right)=\operatorname{Hom}(K, E)$ and so $\operatorname{Hom}\left(\mathbf{F}_{p^{f}}, \overline{\mathbf{F}}_{p}\right)=\operatorname{Hom}\left(\mathbf{F}_{p^{f}}, \mathbf{F}\right)$.

Let $\sigma \in \operatorname{Gal}\left(\mathbf{F}_{p^{f}} / \mathbf{F}_{p}\right)$ be the absolute Frobenius on $\mathbf{F}_{p^{f}}$. We have an isomorphism

$$
\begin{aligned}
\iota: \mathbf{F}_{p^{f}} \otimes_{\mathbf{F}_{p}} \mathbf{F} & \stackrel{\sim}{\rightarrow} \oplus_{\sigma^{j}} \mathbf{F} \\
a \otimes b & \mapsto\left(\sigma^{j}(a) b\right)_{j}
\end{aligned}
$$

and, for $i \in\{0, \ldots, f-1\}$, we write $e_{i} \in \mathbf{F}_{p^{f}} \otimes_{\mathbf{F}_{p}} \mathbf{F}$ to denote the standard idempotent element defined by $\iota\left(e_{i}\right)=\left(\delta_{i, j}\right)_{j}\left(\delta_{i, j}\right.$ being the Kronecker delta function).

The Breuil ring $S$ is defined as the $p$-adic completion of divided power envelope, with respect to the ideal generated by $E(u)$, of the polynomial algebra $W\left(\mathbf{F}_{p^{f}}\right)[u], W\left(\mathbf{F}_{p^{f}}\right)$ being the ring of Witt vectors associated to $\mathbf{F}_{p^{f}}$ (and identified with the ring of integers of $\left.K_{0}\right)$. We write $S_{\mathcal{O}_{E}} \stackrel{\text { def }}{=} S \otimes \mathbf{z}_{p} \mathcal{O}_{E}$ and $\overline{\mathrm{S}}_{\mathbf{F}} \stackrel{\text { def }}{=} S_{\mathcal{O}_{E}} /\left(1 \otimes \varpi, E(u)^{p} \otimes 1\right)$. Concretely, $\overline{\mathrm{S}}_{\mathbf{F}} \cong\left(\mathbf{F}_{p^{f}} \otimes_{\mathbf{F}_{p}} \mathbf{F}\right)[u] / u^{e p}$.

Recall that the ring $S_{\mathcal{O}_{E}}$ is endowed with a residual action of $\operatorname{Gal}\left(K / \mathbf{Q}_{p}\right)$. Concretely, the choice of the uniformizer $\pi \in K$ provides us with a map

$$
\begin{aligned}
\omega_{\pi}: \operatorname{Gal}\left(K / \mathbf{Q}_{p}\right) & \longrightarrow W\left(\mathbf{F}_{p^{f}}\right) \\
g & \longmapsto \frac{g(\pi)}{\pi}
\end{aligned}
$$

and the residual action on the Breuil ring $S_{\mathcal{O}_{E}}$ is uniquely defined (by semilinearity and continuity) via the condition $\widehat{g}(u) \stackrel{\text { def }}{=}\left(\omega_{\pi}(g) \otimes 1\right) u$ for any $g \in \operatorname{Gal}\left(K / \mathbf{Q}_{p}\right)$. This induces an action on $\overline{\mathrm{S}}_{\mathbf{F}}$ by base change in the natural way. For notational convenience, we write $\overline{\mathrm{S}}_{0} \stackrel{\text { def }}{=}\left(\overline{\mathrm{S}}_{\mathbf{F}}\right)^{\operatorname{Gal}\left(K / K_{0}\right)=i d}$, which is naturally identified with $\overline{\mathrm{S}}_{0} \stackrel{\text { def }}{=}\left(\mathbf{F}_{p^{f}} \otimes_{\mathbf{F}_{p}} \mathbf{F}\right)\left[u^{e}\right] / u^{e p}$.

We write $\varepsilon_{p}$ for the $p$-adic cyclotomic character and $\omega$ for its mod $p$ reduction. We convene that $\varepsilon_{p}$ has a Hodge-Tate weight -1 . The choice of an embedding $\mathbf{F}_{p^{f}} \hookrightarrow \mathbf{F}$ provides us with a Serre's fundamental character of niveau $f, \omega_{f}: \operatorname{Gal}\left(K / K_{0}\right) \rightarrow \mathbf{F}$. Note that $\left.\omega_{f} \equiv \omega_{\pi}\right|_{\operatorname{Gal}\left(K / K_{0}\right)}$ modulo $p$.

For a place $w \mid p$ in $F$ we normalize the local reciprocity map $F_{w}^{\times} \rightarrow G_{F_{w}}^{a b}$ in such a way that the inverse of uniformizers are sent to arithmetic Frobenius elements Frob ${ }_{w} \in G_{F_{w}}$. Similarly, we fix an isomorphism $\imath: \overline{\mathbf{Q}}_{p} \stackrel{\sim}{\rightarrow} \mathbf{C}$ and define the local Langlands correspondence $\operatorname{rec}_{F_{w}}$ over $\overline{\mathbf{Q}}_{p}$ by normalizing the classical local correspondence $\operatorname{rec}_{F_{w}, \mathbf{C}}$ over $\mathbf{C}$ ([HT01], [Hen00]) via $\imath \circ \operatorname{rec}_{F_{w}}=\operatorname{rec}_{F_{w}, \mathbf{C}} \circ \imath$.

Given a potentially semistable $p$-adic representation $\rho: G_{\mathbf{Q}_{p}} \rightarrow \mathrm{GL}_{n}(E)$, we write $\operatorname{WD}(\bar{\rho})$ to denote the associated Weil-Deligne representation (cf. [Fon94] and [CDT99], Appendix A) and we refer to $\left.\mathrm{WD}(\rho)\right|_{I_{\mathbf{Q}_{p}}}$ as to the Galois type associated to $\rho$.

We have a contravariant functor $\mathrm{T}_{\mathrm{st}}: \mathbf{F}$ - $\operatorname{BrMod}_{\mathrm{dd}}^{r} \rightarrow \operatorname{Rep}_{\mathbf{F}}\left(G_{\mathbf{Q}_{p}}\right)$, which is exact and faithful. We write $\mathrm{T}_{\mathrm{st}}^{*}$ for the functor $\mathrm{T}_{\mathrm{st}}^{*, r}$ defined as in [EGH13], Definition 3.2.8. In particular, from Section $4, \mathrm{~T}_{\mathrm{st}}^{*}$ always means $\mathrm{T}_{\mathrm{st}}^{*, 2}$.

We write $\ell \ell(\bar{\rho})$ for the Loewy length of $\bar{\rho}: G_{\mathbf{Q}_{p}} \rightarrow \mathrm{GL}_{n}(\mathbf{F})$, i.e., the length of socle filtration of $\bar{\rho}$. We use this notion to measure how far $\bar{\rho}$ is from being semi-simple. We 
say that an ordinary representation $\bar{\rho}$ is maximally non-split if $\ell \ell(\bar{\rho})=n$, and is minimally non-split if $\ell \ell(\bar{\rho})=2$. Note that $\bar{\rho}$ is semi-simple if and only if $\ell \ell(\bar{\rho})=1$.

\section{Integral p-Adic Hodge theory, I: Preliminaries}

The aim of this section is to recall certain categories of semilinear algebraic objects (strongly divisible modules, Breuil-Kisin modules, Fontaine-Laffaille modules) and their relations with Galois representations. None of the results in this section is new and we refer the reader to [HM], Section 2 and [EGH13], Section 3 for more details.

We keep the notations and conventions of Section 1. In particular, we have $e=p^{f}-1$ and $K / K_{0}$ is totally and tamely ramified of degree $e$, the residue field being $k=\mathbf{F}_{p^{f}}$.

Recall that a filtered $\left(\varphi, N, K / \mathbf{Q}_{p}, E\right)$-module of rank $n$ is the datum of a free $K_{0} \otimes \mathbf{Q}_{p} E$ module $D$ of rank $n$ endowed with

- a semilinear automorphism $\varphi$ (semilinear with respect to the absolute Frobenius on $\left.K_{0}\right)$;

○ a nilpotent, $K_{0} \otimes_{\mathbf{Q}_{p}} E$-linear endomorphism $N$ such that $N \varphi=p \varphi N$;

○ a decreasing, exhaustive and separated filtration $\left(\mathrm{Fil}^{i} D_{K}\right)_{i \in \mathbf{Z}}$ on $D_{K} \stackrel{\text { def }}{=} K \otimes_{K_{0}} D$ by $K \otimes \mathbf{Q}_{p} E$-submodules;

- a $\operatorname{Gal}\left(K / \mathbf{Q}_{p}\right)$ action by $E$-linear and $K_{0}$-semilinear automorphisms which are compatible with the additional structures on $D, D_{K}$ (i.e. Frobenius, monodromy and filtration).

We write $\operatorname{Mod}_{E}\left(\varphi, N, K / \mathbf{Q}_{p}\right)$ to denote the category of filtered $\left(\varphi, N, K / \mathbf{Q}_{p}, E\right)$-modules of finite rank and by $\operatorname{Mod}_{E}^{\text {w.a. }}\left(\varphi, N, K / \mathbf{Q}_{p}\right)$ the full sub-category of weakly admissible filtered $\left(\varphi, N, K / \mathbf{Q}_{p}, E\right)$.

We recall (cf. [EGH13], Definition 3.1.1) that we have the contravariant functors $\mathrm{V}_{s t}^{\mathbf{Q}_{p}}$ : $\operatorname{Mod}_{E}^{\text {w.a. }}\left(\varphi, N, K / \mathbf{Q}_{p}\right) \rightarrow \operatorname{Rep}_{E}^{K \text {-st }}\left(G_{\mathbf{Q}_{p}}\right)$ and $\mathrm{D}_{s t}^{\mathbf{Q}_{p}}: \operatorname{Rep}_{E}^{K \text {-st }}\left(G_{\mathbf{Q}_{p}}\right) \rightarrow \operatorname{Mod}_{E}^{\text {w.a. }}\left(\varphi, N, K / \mathbf{Q}_{p}\right)$, establishing an equivalence of categories. (These functors depend on the choice of uniformizers and we choose $p$ as in [EGH13].)

Fix a positive integer $r<p-1$. The category $\mathcal{O}_{E}-\mathfrak{M o d}{ }_{\mathrm{dd}}^{r}$ of strongly divisible $\mathcal{O}_{E}$-modules with descent data is defined to be the category of finitely generated free $S_{\mathcal{O}_{E}}$-modules $\widehat{\mathcal{M}}$ with a sub $S_{\mathcal{O}_{E}}$-module $\operatorname{Fil}^{r} \widehat{\mathcal{M}}$, additive maps $\varphi, N: \widehat{\mathcal{M}} \rightarrow \widehat{\mathcal{M}}$, and $S_{\mathcal{O}_{E}}$-semilinear bijections $\widehat{g}: \widehat{\mathcal{M}} \rightarrow \widehat{\mathcal{M}}$ for each $g \in \operatorname{Gal}\left(K / \mathbf{Q}_{p}\right)$ such that the following conditions hold:

- $\operatorname{Fil}^{r} \widehat{\mathcal{M}}$ contains $\left(\mathrm{Fil}^{r} S_{\mathcal{O}_{E}}\right) \widehat{\mathcal{M}}$

- $\mathrm{Fil}^{r} \widehat{\mathcal{M}} \cap I \widehat{\mathcal{M}}=I \mathrm{Fil}^{r} \widehat{\mathcal{M}}$ for all ideals $I$ of $\mathcal{O}_{E}$;

- $\varphi(s x)=\varphi(s) \varphi(x)$ for all $s \in S_{\mathcal{O}_{E}}$ and $x \in \widehat{\mathcal{M}}$;

- $\varphi\left(\mathrm{Fil}^{r} \widehat{\mathcal{M}}\right)$ is contained in $p^{r} \widehat{\mathcal{M}}$ and generates it over $S_{\mathcal{O}_{E}}$;

○ $N(s x)=N(s) x+s N(x)$ for all $s \in S_{\mathcal{O}_{E}}$ and $x \in \widehat{\mathcal{M}}$;

○ $N \varphi=p \varphi N$;

- $E(u) N\left(\mathrm{Fil}^{r} \widehat{\mathcal{M}}\right) \subset \mathrm{Fil}^{r} \widehat{\mathcal{M}}$;

○ for all $g \in \operatorname{Gal}\left(K / \mathbf{Q}_{p}\right), \widehat{g}$ commutes with $\varphi$ and $N$, and preserves $\operatorname{Fil}^{r} \widehat{\mathcal{M}}$;

$\widehat{\circ g_{1} \circ g_{2}}=\widehat{g}_{1} \circ \widehat{g}_{2}$ for all $g_{1}, g_{2} \in \operatorname{Gal}\left(K / \mathbf{Q}_{p}\right)$.

The morphisms are $S_{\mathcal{O}_{E}}$-module homomorphisms that preserve Fil ${ }^{r} \widehat{\mathcal{M}}$ and commute with $\varphi_{r}, N$, and the descent data action.

We have a functor $\mathrm{T}_{\mathrm{st}}^{\mathbf{Q}_{p}}: \mathcal{O}_{E^{-}} \mathfrak{M o d}_{\mathrm{dd}}^{r} \rightarrow \operatorname{Rep}_{\mathcal{O}_{E}}^{K \text {-st,[-r,0]}}\left(G_{\mathbf{Q}_{p}}\right)$ where $\operatorname{Rep}_{\mathcal{O}_{E}}^{K \text {-st, }[-r, 0]}\left(G_{\mathbf{Q}_{p}}\right)$ is the category of $G_{\mathbf{Q}_{p}}$-stable $\mathcal{O}_{E}$-lattices inside $E$-valued, finite dimensional $p$-adic Galois 
representation of $G_{\mathbf{Q}_{p}}$ becoming semi-stable over $K$ and with Hodge-Tate weights in [-r,0] (cf. [EGH13], Section 3.1).

The following deep theorem provides the link between lattices in potentially semi-stable Galois representations and strongly divisible modules:

Theorem 2.1 ([Liu08], [EGH13]). The functor

$$
\mathrm{T}_{\mathrm{st}}^{\mathbf{Q}_{p}}: \mathcal{O}_{E}-\mathfrak{M N o d}_{\mathrm{dd}}^{r} \rightarrow \operatorname{Rep}_{\mathcal{O}_{E}}^{K-s t,[-r, 0]}\left(G_{\mathbf{Q}_{p}}\right)
$$

establishes an equivalence of categories if $r<p-1$.

Moreover, by letting $\rho \stackrel{\text { def }}{=} \mathrm{T}_{\mathrm{st}}^{\mathbf{Q}_{p}}(\widehat{\mathcal{M}}) \otimes_{\mathcal{O}_{E}} E$, we have an isomorphism

$$
\widehat{\mathcal{M}} \otimes_{S_{\mathcal{O}_{E}}} E \cong D_{s t}^{\mathbf{Q}_{p}}(\rho)
$$

via the base change $S_{\mathcal{O}_{E}} \rightarrow E$ defined by $u \mapsto 0$.

Note that (2) provides the relation between the descent data on $\widehat{\mathcal{M}}$ and the Galois type on $\rho$.

Recall that the category $\mathbf{F}$-BrMod ${ }_{\mathrm{dd}}^{r}$ of Breuil modules of weight $r$ with descent data from $K$ to $\mathbf{Q}_{p}$ and coefficients in $\mathbf{F}$ consists of quintuples $\left(\mathcal{M}, \operatorname{Fil}^{r} \mathcal{M}, \varphi_{r}, \hat{g}, N\right)$ where:

$\circ \mathcal{M}$ is a finitely generated $\left(k \otimes_{\mathbf{F}_{p}} \mathbf{F}\right)[u] / u^{e p}$-module, free over $k[u] / u^{e p}$, (which implies that $\mathcal{M}$ is in fact a free $\left(k \otimes_{\mathbf{F}_{p}} \mathbf{F}\right)[u] / u^{e p}$-module of finite rank);

- $\mathrm{Fil}^{r} \mathcal{M}$ is a $\left(k \otimes_{\mathbf{F}_{p}} \mathbf{F}\right)[u] / u^{e p}$-submodule of $\mathcal{M}$ containing $u^{e r} \mathcal{M}$;

- $\varphi_{r}: \mathrm{Fil}^{r} \mathcal{M} \rightarrow \mathcal{M}$ is $\mathbf{F}$-linear and $\varphi$-semilinear (where $\varphi: k[u] / u^{e p} \rightarrow k[u] / u^{e p}$ is the $p$-th power map) with image generating $\mathcal{M}$ as $\left(k \otimes_{\mathbf{F}_{p}} \mathbf{F}\right)[u] / u^{e p}$-module;

$\circ N: \mathcal{M} \rightarrow \mathcal{M}$ is $k \otimes_{\mathbf{F}_{p}} \mathbf{F}$-linear and satisfies

$-N(u x)=u N(x)-u x$ for all $x \in \mathcal{M}$,

$-u^{e} N\left(\mathrm{Fil}^{r} \mathcal{M}\right) \subset \mathrm{Fil}^{r} \mathcal{M}$, and

- $\varphi_{r}\left(u^{e} N(x)\right)=c N\left(\varphi_{r}(x)\right)$ for all $x \in \mathrm{Fil}^{r} \mathcal{M}$, where $c \in\left(k[u] / u^{e p}\right)^{\times}$is the image of $\frac{1}{p} \varphi(E(u))$ under the natural map $S \rightarrow k[u] / u^{e p}$. (Note that $c=1$ in our case, since we choose $E(u)=u^{e}+p$.)

- $\widehat{g}: \mathcal{M} \rightarrow \mathcal{M}$ is an additive bijection for each $g \in \operatorname{Gal}\left(K / \mathbf{Q}_{p}\right)$, preserving $\operatorname{Fil}^{r} \mathcal{M}$, commuting with $\varphi_{r^{-}}$and $N$-actions, and satisfying $\widehat{g_{1} \circ g_{2}}=\widehat{g}_{1} \circ \widehat{g}_{2}$ for $g_{1}, g_{2} \in$ $\operatorname{Gal}\left(K / \mathbf{Q}_{p}\right)$. Furthermore, $\widehat{g}\left(a u^{i} m\right)=g(a)\left((g(\pi) \pi)^{i} \otimes 1\right) u^{i} \widehat{g}(m)$ for $a \in k \otimes_{\mathbf{F}_{p}} \mathbf{F}$ and $m \in \mathcal{M}$.

The morphisms are $\left(k \otimes_{\mathbf{F}_{p}} \mathbf{F}\right)[u] / u^{e p}$-module homomorphisms that preserve $\mathrm{Fil}^{r}$ and commute with $\varphi_{r}, N$, and the descent data action.

As for strongly divisible modules, we have a contravariant functor $\mathrm{T}_{\mathrm{st}}: \mathbf{F}-\mathrm{BrMod}_{\mathrm{dd}}^{r} \rightarrow$ $\operatorname{Rep}_{\mathbf{F}}\left(G_{\mathbf{Q}_{p}}\right)$, which is exact and faithful. We write $\mathrm{T}_{\mathrm{st}}^{*}$ for the functor $\mathrm{T}_{\mathrm{st}}^{*, r}$ defined as in [EGH13], Definition 3.2.8. Recall that we have a base change morphism $S_{\mathcal{O}_{E}} \rightarrow \overline{\mathrm{S}}_{\mathbf{F}}$; the compatibility between the functors $\mathrm{T}_{\mathrm{st}}^{\mathbf{Q}_{p}}$ and $\mathrm{T}_{\mathrm{st}}$ is described by the following

Proposition 2.2. Let $\widehat{\mathcal{M}}$ be an object in $\mathcal{O}_{E}-\mathfrak{M M o d}_{\mathrm{dd}}^{r}$. Then $\widehat{\mathcal{M}} \otimes_{S_{\mathcal{O}_{E}}} \overline{\mathrm{S}}_{\mathbf{F}}$ is naturally an object in $\mathbf{F}$-BrMod ${ }_{\mathrm{dd}}^{r}$.

Moreover the following compatibility is satisfied:

$$
\mathrm{T}_{\mathrm{st}}^{\mathbf{Q}_{p}}(\widehat{\mathcal{M}}) \otimes_{\mathcal{O}_{E}} \mathbf{F} \cong \mathrm{T}_{\mathrm{st}}\left(\widehat{\mathcal{M}} \otimes_{S_{\mathcal{O}_{E}}} \overline{\mathrm{S}}_{\mathbf{F}}\right) .
$$

Proof. This is well known. See for instance [HM], Proposition 2.2.14.

The category $\mathbf{F}-\mathcal{F} \mathcal{L}$ of Fontaine-Laffaille modules consists of triple $\left(M\right.$, Fil $\left.{ }^{\bullet} M, \varphi_{\bullet}\right)$, where 
○ $M$ is a free $k \otimes_{\mathbf{F}_{p}} \mathbf{F}$-module of finite rank;

$\circ\left\{\mathrm{Fil}^{j} M\right\}_{j \in \mathbf{Z}}$ is a separated, exhaustive and decreasing filtration on $M$ by $k \otimes \mathbf{F}_{p} \mathbf{F}$ submodules;

$\circ$ for each $j \in \mathbf{Z}, \varphi_{j}: \mathrm{Fil}^{j} M \rightarrow M$ is a $\varphi$-semilinear Frobenius morphism such that $\mathrm{Fil}^{j+1} M \subset \operatorname{Ker}\left(\varphi_{j}\right)$. Moreover, $\underset{i \in \mathbf{Z}}{\oplus} \varphi_{j}: \underset{j \in \mathbf{Z}}{\oplus} \mathrm{Fil}^{j} M \rightarrow M$ is surjective.

The morphisms are defined in the obvious way.

As for the case of Breuil modules, we have a contravariant functor $T_{\text {cris }}: \mathbf{F}-\mathcal{F} \mathcal{L} \rightarrow$ $\operatorname{Rep}_{\mathbf{F}}\left(G_{\mathbf{Q}_{p}}\right)$, which is exact and fully faithful (cf. [FL82], Section 6.1). We say a representation $\bar{\rho}: G_{\mathbf{Q}_{p}} \rightarrow \mathrm{GL}_{n}(\mathbf{F})$ is Fontaine-Laffaille if it arises from a Fontaine-Laffaille module under the functor $T_{\text {cris }}$.

Before stating the main comparison theorem, we still need to recall the following categories of étale $\varphi$-modules, first introduced by Fontaine ([Fon90]).

Let $k((\underline{p}))$ be the field of norms associated to $\left(K_{0}, p\right)$. In particular, $\underline{p}$ is identified with a sequence $\left(p_{n}\right)_{n} \in\left(\overline{\mathbf{Q}}_{p}\right)^{\mathbf{N}}$ verifying $p_{n}^{p}=p_{n-1}$ for all $n$. We define the category $\left(\varphi, \mathbf{F} \otimes \mathbf{F}_{p} k((\underline{p}))\right)$-Mod of étale $\left(\varphi, \mathbf{F} \otimes_{\mathbf{F}_{p}} k((\underline{p}))\right)$-modules as the category of free $\mathbf{F} \otimes_{\mathbf{F}_{p}}$ $k((\underline{p}))$-modules of finite rank $\mathfrak{D}$ endowed with a semilinear map $\varphi: \mathfrak{D} \rightarrow \mathfrak{D}$ (semilinear with respect to the Frobenius on $k((p))$ ) and inducing an isomorphism $\varphi^{*} \mathfrak{D} \rightarrow \mathfrak{D}$ (with obvious morphisms between objects).

By work of Fontaine [Fon90], we have an anti-equivalence

$$
\begin{aligned}
\left(\varphi, \mathbf{F} \otimes \mathbf{F}_{p} k((\underline{p}))\right)-\mathfrak{M o d} & \stackrel{\sim}{\longrightarrow} \operatorname{Rep}_{\mathbf{F}}\left(G_{\left(K_{0}\right)_{\infty}}\right) \\
\mathfrak{D} & \longmapsto \operatorname{Hom}\left(\mathfrak{D}, k((\underline{p}))^{\mathrm{sep}}\right) .
\end{aligned}
$$

Let us consider $\pi \stackrel{\text { def }}{=} \sqrt[e]{-p} \in K$. We can fix a sequence $\left(\pi_{n}\right)_{n} \in\left(\overline{\mathbf{Q}}_{p}\right)^{\mathbf{N}}$ such that $\pi_{n}^{e}=p_{n}$ for all $n \in \mathbf{N}$ and which is compatible with the norm maps $K\left(\pi_{n+1}\right) \rightarrow K\left(\pi_{n}\right)$ (cf. [Bre], Appendix A).

By letting $K_{\infty} \stackrel{\text { def }}{=} \cup_{n \in \mathbf{N}} K_{n}$ (where we let $\left.K_{n} \stackrel{\text { def }}{=} K\left(\pi_{n}\right)\right)$ and $\left(K_{0}\right)_{\infty} \stackrel{\text { def }}{=} \cup_{n \in \mathbf{N}} K_{0}\left(p_{n}\right)$, we have a canonical isomorphism $\operatorname{Gal}\left(K_{\infty} /\left(K_{0}\right)_{\infty}\right) \rightarrow \operatorname{Gal}\left(K / K_{0}\right)$ and we will identify $\omega_{\pi}$ as a character on $\operatorname{Gal}\left(K_{\infty} /\left(K_{0}\right)_{\infty}\right)$.

The field of norms $k((\underline{\pi}))$ associated to $(K, \pi)$ is then endowed with a residual action of $\operatorname{Gal}\left(K_{\infty} /\left(K_{0}\right)_{\infty}\right)$, which is completely determined by $\widehat{g}(\underline{\pi})=\omega_{\pi}(g) \underline{\pi}$.

We can therefore define the category $\left(\varphi, \mathbf{F} \otimes_{\mathbf{F}_{p}} k((\underline{\pi}))\right)-\mathfrak{M o d}_{\mathrm{dd}}$ of étale $\left(\varphi, \mathbf{F} \otimes_{\mathbf{F}_{p}} k((\underline{\pi}))\right)$ modules with descent data: an object $\mathfrak{D}$ is defined in the analogous, evident way as for the category $\left(\varphi, \mathbf{F} \otimes_{\mathbf{F}_{p}} k((p))\right)$-Modo, but we moreover require that $\mathfrak{D}$ is endowed with a semilinear action of $\operatorname{Gal}\left(K_{\infty} /\left(K_{0}\right)_{\infty}\right)$ (semilinear with respect to the residual action on $\mathbf{F} \otimes \mathbf{F}_{p} k((\underline{\pi}))$, where $\mathbf{F}$ is endowed with the trivial $\operatorname{Gal}\left(K_{\infty} /\left(K_{0}\right)_{\infty}\right)$-action) and the Frobenius $\varphi$ is $\operatorname{Gal}\left(K_{\infty} /\left(K_{0}\right)_{\infty}\right)$-equivariant.

From $[\mathrm{HM}]$, Appendix 5.2 (which builds on the classical result of Fontaine) we have an anti-equivalence

$$
\begin{aligned}
\left(\varphi, \mathbf{F} \otimes_{\mathbf{F}_{p}} k((\underline{\pi}))\right)-\mathfrak{M o d}_{\mathrm{dd}} & \stackrel{\sim}{\longrightarrow} \operatorname{Rep}_{\mathbf{F}}\left(G_{\left.\left(K_{0}\right)_{\infty}\right)}\right) \\
\mathfrak{D} & \mapsto \operatorname{Hom}\left(\mathfrak{D}, k((\underline{\pi}))^{\mathrm{sep}}\right) .
\end{aligned}
$$

The main result concerning the relations between the various categories and functors introduced so far is summarized by the following proposition ([HM], Proposition 2.3.1) 
Proposition 2.3. There exist faithful functors

$$
M_{k((\underline{\pi}))}: \mathbf{F}-\operatorname{BrMod}_{\mathrm{dd}}^{r} \rightarrow\left(\varphi, \mathbf{F} \otimes \mathbf{F}_{p} k((\underline{\pi}))\right)-\mathfrak{M}_{\mathfrak{m} \mathfrak{d}_{\mathrm{dd}}}
$$

and

$$
\mathcal{F}: \mathbf{F}-\mathcal{F} \mathcal{L}^{[0, p-2]} \rightarrow\left(\varphi, \mathbf{F} \otimes_{\mathbf{F}_{p}} k((\underline{p}))\right)-\mathfrak{M o d}
$$

fitting in the following commutative diagram:

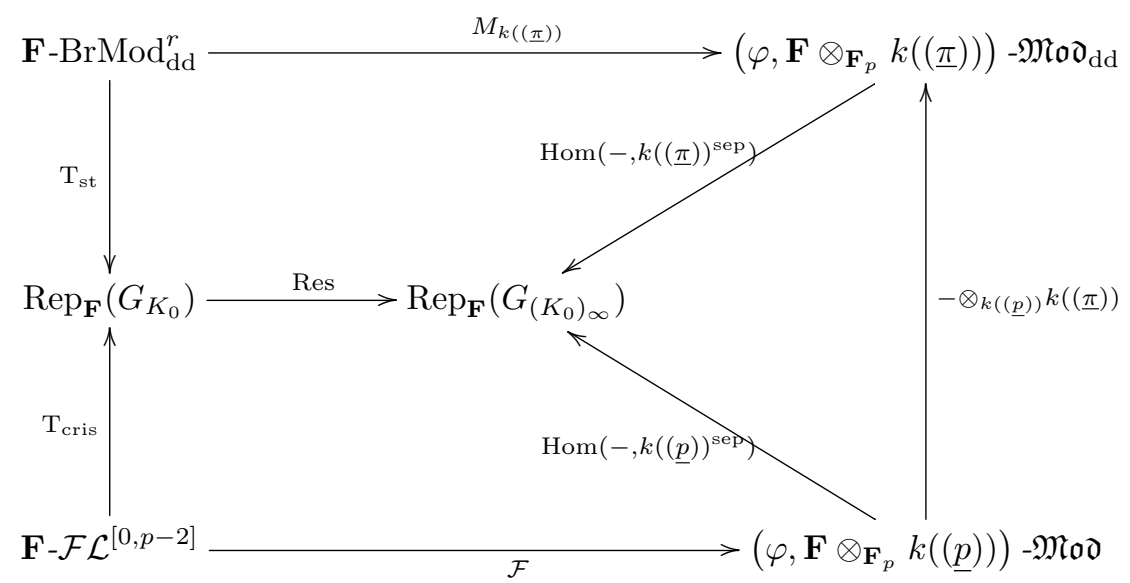

where the descent data is relative to $K_{0}$ and the functor Res $\circ \mathrm{T}_{\text {cris }}$ is fully faithful.

The functors $M_{k((\underline{\pi}))}, \mathcal{F}$ are defined in [HM], Appendix A, building on the classical work of Breuil [Bre99] and Caruso-Liu [CL09]. In certain cases, the description of the functor $M_{k((\underline{\pi}))}$ is particularly concrete.

Assume that the Breuil module $\mathcal{M}$ has rank $n$, with descent data associated to a Galois type $\tau: I_{\mathbf{Q}_{p}} \rightarrow \mathrm{GL}_{n}\left(\mathcal{O}_{E}\right)$. By writing $\tau=\left[\omega_{f}\right]^{a_{1}} \oplus \cdots \oplus\left[\omega_{f}\right]^{a_{n}}$ we can find a basis $\left(e_{1}, \ldots, e_{n}\right)$ for $\mathcal{M}$ and a system of generators $\left(f_{1}, \ldots, f_{n}\right)$ for $\mathrm{Fil}^{r} \mathcal{M}$ which are compatible with $\tau$ :

$$
\widehat{g} \cdot e_{i}=\left(\omega_{f}^{a_{i}}(g) \otimes 1\right) e_{i}, \quad \widehat{g} \cdot f_{i}=\left(\omega_{f}^{a_{i}}(g) \otimes 1\right) f_{i}
$$

for all $i=1, \ldots, n$ and all $g \in \operatorname{Gal}\left(K / K_{0}\right)$ (cf. [HM], Section 2.3.2).

In this case we say that the basis $\underline{e}$ and the system of generators $\underline{f}$ are compatible with the Galois type $\tau$.

Lemma 2.4. Let $\mathcal{M}$ be a Breuil module of rank n, with descent data associated to a Galois type $\tau: I_{\mathbf{Q}_{p}} \rightarrow \mathrm{GL}_{n}\left(\mathcal{O}_{E}\right)$ and let $\underline{e}, \underline{f}$ be a basis for $\mathcal{M}$ and a system of generators for Fil $^{r} \mathcal{M}$ respectively, which are moreover compatible with $\tau$.

Write $V=V_{\underline{e}, \underline{f}} \in M_{n}\left(\overline{\mathrm{S}}_{\mathbf{F}}\right)$ for the matrix giving the coordinates of $\underline{f}$ in the basis $\underline{e}$ and $A \stackrel{\text { def }}{=} \mathrm{Mat}_{\underline{e}, \underline{f}}\left(\varphi_{r}\right) \in \mathrm{GL}_{n}\left(\overline{\mathrm{S}}_{\mathbf{F}}\right)$ for the matrix describing the Frobenius action on $\mathcal{M}$ with respect to $\underline{e}, \underline{f}$.

Then there exists a basis $\mathfrak{e}$ for $M_{k((\underline{\pi}))}\left(\mathcal{M}^{*}\right)$, compatible with the descent data, such that the Frobenius action is described by

$$
\operatorname{Mat}_{\underline{\mathfrak{e}}}(\phi)=\widehat{V}^{t}\left(\widehat{A}^{-1}\right)^{t} \in M_{n}\left(\mathbf{F} \otimes_{\mathbf{F}_{p}} k((\underline{\pi}))\right)
$$

where $\widehat{V}$, $\widehat{A}$ are lifts of $V, A$ in $M_{n}\left(\mathbf{F} \otimes_{\mathbf{F}_{p}} k((\underline{\pi}))\right)$ via the reduction morphism $\mathbf{F} \otimes_{\mathbf{F}_{p}} k((\underline{\pi})) \rightarrow$ $\overline{\mathrm{S}}_{\mathbf{F}}$.

Proof. This is $[\mathrm{HM}]$, Lemma 2.3.5. We just recall that $\mathcal{M}^{*}$ denotes the dual Breuil module as defined in [EGH13], Definition 3.2.8. 


\section{Integral $p$-ADiC Hodge TheORY, II: COMPlements}

This section is the technical heart of the paper. We describe rank one Breuil modules with tame descent data, and the extensions between them. The main result is Lemma 3.2, often referred as the splitting lemma.

We keep the notations as in the previous sections. In particular, we fix integers $f \geq 1$, $0 \leq r<p-1$, letting $K_{0} \stackrel{\text { def }}{=} \mathbf{Q}_{p^{f}}$ be the unramified extension of $\mathbf{Q}_{p}$ of degree $f$ and $K \stackrel{\text { def }}{=} K_{0}(\sqrt[e]{-p})$ with $e=p^{f}-1$.

Lemma 3.1. Let $\mathcal{M}$ be a rank-one object in $\mathbf{F}-\operatorname{BrMod}_{\mathrm{dd}}^{r}$ with niveau $f$ descent data relative to $\mathbf{Q}_{p}$. Then there exists a generator $m \in \mathcal{M}$ such that:

(i) $\mathcal{M}=\overline{\mathrm{S}}_{\mathbf{F}} \cdot m$;

(ii) $\operatorname{Fil}^{r} \mathcal{M}=u^{s(p-1)} \mathcal{M}$ where $0 \leq s \leq \frac{r e}{p-1}$;

(iii) $\varphi_{r}\left(u^{s(p-1)}\right)=\lambda m$ for some $\lambda \in\left(\mathbf{F}_{p^{f}} \otimes \mathbf{F}_{p} \mathbf{F}\right)^{\times}$;

(iv) $\widehat{g}(m)=\left(\omega_{f}(g)^{k} \otimes 1\right) m$ for all $g \in \operatorname{Gal}\left(K / K_{0}\right)$ where $k$ is an integer such that $k+p s \equiv 0 \bmod \frac{e}{p-1}$

(v) $N(m)=0$.

Moreover, one has

$$
\left.\mathrm{T}_{\mathrm{st}}^{*}(\mathcal{M})\right|_{I_{\mathbf{Q}_{p}}}=\omega_{f}^{k+p s} .
$$

We denote the Breuil modules in the preceding lemma by $\mathcal{M}(k, s, \lambda)$.

Proof. Recall from $\S 1$ the idempotent elements $e_{i} \in \mathbf{F}_{p^{f}} \otimes_{\mathbf{F}_{p}} \mathbf{F}$. From the Lemma 3.3.2 in [EGH13], it is known that every rank-one Breuil module $\mathcal{M}$ with tame descent data from $K$ to $K_{0}$ has a generator $m$ such that $\mathcal{M}=\overline{\mathrm{S}}_{\mathbf{F}} \cdot m, e_{i} \operatorname{Fil}^{r} \mathcal{M}=u^{r_{i}} e_{i} \mathcal{M}, \varphi_{r}\left(\sum_{i=0}^{f-1} u^{r_{i}} e_{i} m\right)=\lambda m$ for some $\lambda \in\left(\mathbf{F}_{p^{f}} \otimes_{\mathbf{F}_{p}} \mathbf{F}\right)^{\times}, \hat{g}(m)=\sum_{i=0}^{f-1}\left(\omega_{f}^{k_{i}}(g) \otimes 1\right) e_{i} m$ for all $g \in \operatorname{Gal}\left(K / K_{0}\right)$, and $N(m)=0$, where $0 \leq r_{i} \leq r e$ and $k_{i} \equiv p\left(k_{i-1}+r_{i-1}\right) \bmod e$ for all $i$.

We let $\varphi \in \operatorname{Gal}\left(K / \mathbf{Q}_{p}\right)$ be the unique lift of the absolute Frobenius $\sigma \in \operatorname{Gal}\left(K_{0} / \mathbf{Q}_{p}\right)$ verifying $\varphi\left(\pi_{K}\right)=\pi_{K}$ (as in the notations of $\S 1$ ). Since $\varphi g \varphi^{-1}=g^{p}$ for all $g \in \operatorname{Gal}\left(K / K_{0}\right)$, we have

$$
\sum_{i=0}^{f-1}\left(\omega_{f}^{p k_{i}}(g) \otimes 1\right) e_{i-1} \varphi(m)=\hat{\varphi} \circ \hat{g}(m)=\hat{g}^{p} \circ \hat{\varphi}(m)=\sum_{i=0}^{f-1}\left(\omega_{f}^{p k_{i}}(g) \otimes 1\right) e_{i} \varphi(m)
$$

and so $k_{0} \equiv k_{1} \equiv \ldots \equiv k_{f-1} \bmod e$. Similarly,

$$
\varphi\left(e_{i} \mathrm{Fil}^{r} \mathcal{M}\right)=u^{r_{i}} e_{i-1} \mathcal{M} \in e_{i-1} \mathrm{Fil}^{r} \mathcal{M}
$$

and so $r_{i} \geq r_{i-1}$ for all $i$. Hence, we conclude that $r_{0}=r_{1}=\ldots=r_{f-1}$. It is now immediate from $k_{i} \equiv p\left(k_{i-1}+r_{i-1}\right) \bmod e$ that $r_{0}=(p-1) s$ for some integer $s$ and $k_{0}+p s \equiv 0 \bmod$ $\frac{e}{p-1}$. Finally, $\left.\mathrm{T}_{\mathrm{st}}^{*}(\mathcal{M})\right|_{I_{\mathbf{Q}_{p}}}=\omega_{f}^{k_{0}+p s}$ by the Lemma 3.3.2 in [EGH13] again.

We are now able to prove the main result of this section, the splitting lemma. In what follows, for an element $m \in \mathbf{Z}$ we define $[m]_{f} \in\{0, \ldots, e-1\}$ via $[m]_{f} \equiv m \bmod e$.

Lemma 3.2. Let $\mathcal{M}_{x}, \mathcal{M}_{y}$ be rank-one objects in $\mathbf{F}$-BrMod ${ }_{\mathrm{dd}}^{r}$ with tame descent data from $K$ to $\mathbf{Q}_{p}$. Assume that the Galois types on $\mathcal{M}_{x}$ and on $\mathcal{M}_{y}$ are $\omega_{f}^{k_{x}}$ and $\omega_{f}^{k_{y}}$ respectively and that $\mathrm{Fil}^{r} \mathcal{M}_{x}=u^{s_{x}(p-1)} \mathcal{M}_{x}$ and $\mathrm{Fil}^{r} \mathcal{M}_{y}=u^{s_{y}(p-1)} \mathcal{M}_{y}$ where the integers $k_{x}, k_{y}, s_{x}, s_{y} \in \mathbf{Z}$ satisfy

$$
p\left(s_{y}-s_{x}\right)+\left[k_{y}-k_{x}\right]_{f}>0 .
$$


Assume further that $f<p$ and let

$$
0 \rightarrow \mathcal{M}_{x} \rightarrow \mathcal{M} \rightarrow \mathcal{M}_{y} \rightarrow 0
$$

be an extension in $\mathbf{F}$-BrMod ${ }_{\mathrm{dd}}^{r}$, with $\mathrm{T}_{\mathrm{st}}^{*}(\mathcal{M})$ being Fontaine-Laffaille.

If the exact sequence of $\overline{\mathrm{S}}_{\mathbf{F}}$-modules

$$
0 \rightarrow \operatorname{Fil}^{r} \mathcal{M}_{x} \rightarrow \mathrm{Fil}^{r} \mathcal{M} \rightarrow \mathrm{Fil}^{r} \mathcal{M}_{y} \rightarrow 0
$$

splits, then the $G_{\mathbf{Q}_{p}}$-representation $\mathrm{T}_{\mathrm{st}}^{*}(\mathcal{M})$ splits as a direct sum of two characters.

In particular, provided that $p k_{y} \not \equiv k_{x}$ modulo $e$ and that $s_{y}(p-1)<$ re if $f>1$, the representation $\mathrm{T}_{\mathrm{st}}^{*}(\mathcal{M})$ splits as a direct sum of two characters if the element $j_{0} \in \mathbf{Z}$ uniquely defined by

$$
j_{0} e+\left[p^{-1} k_{y}-k_{x}\right]_{f}<s_{x}(p-1) \leq\left(j_{0}+1\right) e+\left[p^{-1} k_{y}-k_{x}\right]_{f}
$$

satisfies

$$
\left(r+j_{0}\right) e+\left[p^{-1} k_{y}-k_{x}\right]_{f}<\left(s_{x}+s_{y}\right)(p-1) .
$$

Proof. Let us fix a basis $\underline{e} \stackrel{\text { def }}{=}\left(e_{x}, e_{y}\right)$ on $\mathcal{M}$ which is compatible with the submodule structure on $\mathcal{M}$ and with the inertial descent data. In other words, $e_{x}$ is a basis for $\mathcal{M}_{x}, e_{y}$ maps to a basis for $\mathcal{M}_{y}$, and $e_{x}$ and $e_{y}$ are eigenvectors for the inertial descent data with $\omega_{f}^{k_{x}}$ and $\omega_{f}^{k_{y}}$ as associated eigencharacters.

Assume that the exact sequence (4) splits. With respect to the basis $\underline{e}$, the filtration and the Frobenius map are then described as follows:

$$
V_{0} \stackrel{\text { def }}{=} \operatorname{Mat}_{\underline{e}}\left(\mathrm{Fil}^{r} \mathcal{M}\right)=\left[\begin{array}{cc}
u^{s_{x}(p-1)} & 0 \\
0 & u^{s_{y}(p-1)}
\end{array}\right]
$$

and

$$
A_{0} \stackrel{\text { def }}{=} \operatorname{Mat}_{\underline{e}, \underline{f}}\left(\varphi_{r}\right)=\left[\begin{array}{cc}
\alpha_{0} & u^{\left[k_{y}-k_{x}\right]_{f}} \gamma_{0} \\
0 & \beta_{0}
\end{array}\right]
$$

where $\alpha_{0}, \beta_{0} \in \overline{\mathrm{S}}_{0}^{\times}$and $\gamma_{0} \in \overline{\mathrm{S}}_{0}$ and where $\underline{f} \stackrel{\text { def }}{=}\left(f_{x}, f_{y}\right)=\left(u^{s_{x}(p-1)} e_{x}, u^{s_{y}(p-1)} e_{y}\right)$.

Set $\underline{e^{\prime}} \stackrel{\text { def }}{=} \underline{e} \cdot A_{0}$. It is a basis for $\mathcal{M}$, formed by eigenvectors for the inertial descent data and compatible with the submodule structure on $\mathcal{M}$. We let

$$
V_{1}=\left[\begin{array}{cc}
u^{s_{x}(p-1)} & 0 \\
0 & u^{s_{y}(p-1)}
\end{array}\right] \text { and } B_{1}=\left[\begin{array}{cc}
\alpha_{0} & u^{\left[p^{-1}\left(k_{y}-k_{x}\right)\right]_{f}} \eta_{1} \\
0 & \beta_{0}
\end{array}\right]
$$

where $u^{\left[p^{-1}\left(k_{y}-k_{x}\right)\right]_{f}} \eta_{1} \in \overline{\mathrm{S}}_{\mathbf{F}}$. Then

$$
A_{0} V_{1}=V_{0} B_{1}
$$

if and only if the condition

$$
\gamma_{0} u^{s_{y}(p-1)+\left[k_{y}-k_{x}\right]_{f}}=\eta_{1} u^{s_{x}(p-1)+\left[p^{-1}\left(k_{y}-k_{x}\right)\right]_{f}}
$$

holds. Since $\left(s_{y}-s_{x}\right)(p-1)+\left[k_{y}-k_{x}\right]_{f}>0$ (which is immediately deduced by $p\left(s_{y}-s_{x}\right)+$ $\left[k_{y}-k_{x}\right]_{f}>0$ ), the equation (6) makes sense and one easily sees that $\underline{e}^{\prime} V_{1}$ forms a system of $\overline{\mathrm{S}}_{\mathbf{F}}$-generators for $\mathrm{Fil}^{r} \mathcal{M}$. Hence, we have

$$
\operatorname{Mat}_{\underline{e}^{\prime}}\left(\mathrm{Fil}^{r} \mathcal{M}\right)=V_{1} \text {. }
$$

Let $\underline{f^{\prime}} \stackrel{\text { def }}{=}\left(f_{x}^{\prime}, f_{y}^{\prime}\right)=V_{1}$. Then

$$
\operatorname{Mat}_{\underline{\underline{\prime}}^{\prime}, \underline{f}^{\prime}}\left(\varphi_{r}\right)=\varphi\left(B_{1}\right),
$$


by equation (6). By equation (7) and noticing that $\gamma_{1} \in \overline{\mathrm{S}}_{0}$ we see that the off diagonal entry of $\varphi\left(B_{1}\right)$ has the form $u^{\left[k_{y}-k_{x}\right]_{f}} \gamma_{1}$ where

$$
\gamma_{1} \stackrel{\text { def }}{=} u^{(p-1)\left[p\left(s_{y}-s_{x}\right)+\left[k_{y}-k_{x}\right]_{f}\right]} \varphi\left(\gamma_{0}\right)
$$

As $p\left(s_{y}-s_{x}\right)+\left[k_{y}-k_{x}\right]_{f}>0$, we can iterate the previous procedure to end up with a basis $\underline{e}^{\prime \prime}$ for $\mathcal{M}$ (formed by eigenvectors for the residual inertial action and compatible with the submodule structure on $\mathcal{M}$ ) such that

$$
\operatorname{Mat}_{\underline{e}^{\prime \prime}}\left(\operatorname{Fil}^{r} \mathcal{M}\right)=\left[\begin{array}{cc}
u^{s_{x}(p-1)} & 0 \\
0 & u^{s_{y}(p-1)}
\end{array}\right] \text { and } \operatorname{Mat}_{\underline{e}^{\prime \prime}, \underline{f}^{\prime \prime}}\left(\varphi_{r}\right)=\left[\begin{array}{cc}
\lambda & 0 \\
0 & \mu
\end{array}\right]
$$

where $\lambda, \mu \in\left(\mathbf{F}_{p^{f}} \otimes_{\mathbf{F}_{p}} \mathbf{F}\right)^{\times}$and $\underline{f}^{\prime \prime}$ is determined by the column vectors of $\operatorname{Mat}_{\underline{e}^{\prime \prime}}\left(\mathrm{Fil}^{r} \mathcal{M}\right)$.

It is now easy to verify that the $\phi$-module (over $\mathbf{F}_{p^{f}} \otimes_{\mathbf{F}_{p}} \mathbf{F}((\underline{\pi}))$ ) defined by $\mathfrak{M} \stackrel{\text { def }}{=}$ $M_{\mathbf{F}_{p f}((\underline{\pi}))}\left(\mathcal{M}^{*}\right)$ (cf. Propostion 2.3 and Lemma 2.4) is described by

$$
\operatorname{Mat}(\phi)=\left[\begin{array}{cc}
\underline{\pi}^{s_{x}(p-1)} \lambda^{-1} & 0 \\
0 & \underline{\pi}^{s_{y}(p-1)} \mu^{-1}
\end{array}\right]
$$

in an appropriate basis $\mathfrak{e}=\left(\mathfrak{e}_{x}, \mathfrak{e}_{y}\right)$ formed by $\left(\omega_{f}^{-p^{-1} k_{x}}, \omega_{f}^{-p^{-1} k_{y}}\right)$-eigenvectors for the descent data. By considering the change of basis $\mathfrak{e}^{\prime}=\left(\underline{\pi}^{p^{f-1} k_{x}} \mathfrak{e}_{x}, \underline{\pi}^{p^{f-1} k_{y}} \mathfrak{e}_{y}\right)$ we have:

$$
\begin{aligned}
\operatorname{Mat}(\phi) & =\left[\begin{array}{cc}
\underline{\pi}^{-p^{f-1} k_{x}} & 0 \\
0 & \underline{\pi}^{-p^{f-1} k_{y}}
\end{array}\right]\left[\begin{array}{cc}
\underline{\pi}^{s_{x}(p-1)} \lambda^{-1} & 0 \\
0 & \underline{\pi}^{s_{y}(p-1)} \mu^{-1}
\end{array}\right]\left[\begin{array}{cc}
\underline{\pi}^{p^{f} k_{x}} & 0 \\
0 & \underline{\pi}^{p^{f} k_{y}}
\end{array}\right] \\
& =\left[\begin{array}{cc}
\underline{\pi}^{(p-1)\left(s_{x}+p^{f-1} k_{x}\right)} \lambda^{-1} & 0 \\
0 & \underline{\pi}^{(p-1)\left(s_{y}+p^{f-1} k_{y}\right)} \mu^{-1}
\end{array}\right] .
\end{aligned}
$$

As $s_{x}+p^{f-1} k_{x}=j_{x} \frac{e}{p-1}$ and $s_{y}+p^{f-1} k_{y}=j_{y} \frac{e}{p-1}$ for some $j_{x}, j_{y} \in \mathbf{Z}$, we see that the $\phi$-module $\mathfrak{M}$ is the base change via $\mathbf{F}_{p^{f}} \otimes_{\mathbf{F}_{p}} \mathbf{F}((\underline{p})) \rightarrow \mathbf{F}_{p^{f}} \otimes_{\mathbf{F}_{p}} \mathbf{F}((\underline{\pi}))$ of the $\phi$-module $\mathfrak{M}_{0}$ (over $\mathbf{F}_{p^{f}} \otimes_{\mathbf{F}_{p}} \mathbf{F}((\underline{p}))$ ) described by

$$
\operatorname{Mat}(\phi)=\left[\begin{array}{cc}
\underline{p}^{j_{x}} \lambda^{-1} & 0 \\
0 & \underline{p}^{j_{y}} \mu^{-1}
\end{array}\right]
$$

In particular, the representation $\operatorname{Hom}\left(\mathfrak{M}_{0}, \mathbf{F}((\underline{p}))^{\text {sep }}\right)$ splits as a direct sum of two characters. As $\mathrm{T}_{\mathrm{st}}^{*}(\mathcal{M})$ is Fontaine-Laffaille, we deduce $\left.\mathrm{T}_{\mathrm{st}}^{*}(\mathcal{M})\right|_{G_{\left(K_{0}\right)_{\infty}}} \cong \operatorname{Hom}\left(\mathfrak{M}_{0}, \mathbf{F}((\underline{p}))^{\text {sep }}\right)$, hence $\left.\mathrm{T}_{\mathrm{st}}^{*}(\mathcal{M})\right|_{G_{K_{0}}}$ splits as a direct sum of two characters via Proposition 2.3. As $p>f$ this implies that $\mathrm{T}_{\mathrm{st}}^{*}(\mathcal{M})$ splits, as required.

Assume now that $s_{x}, s_{y}, k_{x}, k_{y}$ satisfy the condition in the final statement of the lemma. In terms of coordinates associated to the basis $\left(e_{x}, e_{y}\right)$ we have

$$
\operatorname{Fil}^{r} \mathcal{M}=\langle\underbrace{\left[\begin{array}{c}
u^{s_{x}(p-1)} \\
0
\end{array}\right]}_{\stackrel{\text { def }}{=} f_{x}}, \underbrace{\left[\begin{array}{c}
u^{\left[p^{-1} k_{y}-k_{x}\right]_{f}} \\
u_{y}^{s_{y}(p-1)}
\end{array}\right]}_{\underline{\text { def }} f_{y}}\rangle+u^{r e} \mathcal{M}
$$

where $v_{0} \in \overline{\mathrm{S}}_{0}$. We let $j \stackrel{\text { def }}{=} \frac{\operatorname{ord}\left(v_{0}\right)}{e}$.

If $j>j_{0}$ then there exists $P(u) \in \overline{\mathrm{S}}_{\mathbf{F}}$ such that $f_{y}-P(u) f_{x}=\left[\begin{array}{c}0 \\ u^{s_{y}(p-1)}\end{array}\right]$. Therefore, the sequence (4) splits and we are in the previous case. 
Assume now that $j \leq j_{0}$ and let us write $v_{0}=\eta u^{j e}+v_{0}^{\prime}$ where $\eta \in\left(\mathbf{F}_{p^{f}} \otimes_{\mathbf{F}_{p}} \mathbf{F}\right) \backslash\{0\}$ and $v_{0}^{\prime} \in u^{(j+1) e} \overline{\mathrm{S}}_{0}$. We claim that $\eta \in\left(\mathbf{F}_{p^{f}} \otimes_{\mathbf{F}_{p}} \mathbf{F}\right)^{\times}$. Indeed, for any $g \in \operatorname{Gal}\left(K / \mathbf{Q}_{p}\right)$ we have $\widehat{g}\left(f_{y}\right)=a \cdot g(\eta) \cdot u^{\left[p^{-1} k_{y}-k_{x}\right]_{f}+j e} e_{x}+b \cdot u^{s_{y}(p-1)} e_{y}+c \cdot e_{x}$ where $a, b \in \overline{\mathrm{S}}_{\mathbf{F}}^{\times}$and $c \in u^{\left[p^{-1} k_{y}-k_{x}\right]_{f}+(j+1) e} \overline{\mathrm{S}}_{\mathbf{F}}(a, b, c$ depending on $g)$. On the other hand, since $f_{x}$ and $f_{y}$ generate Fil ${ }^{r} \mathcal{M}$ modulo $u^{r e} \mathcal{M}$, we have $\widehat{g}\left(f_{y}\right)=\alpha \cdot f_{x}+\beta \cdot f_{y}+\gamma \cdot u^{r e} e_{y}$ (again, $\alpha, \beta, \gamma$ depending on $g$ ). By looking at the $e_{y}$-component in $\alpha \cdot f_{x}+\beta \cdot f_{y}+\gamma \cdot u^{r e} e_{y}$ and since $s_{y}(p-1)<r e$ we easily see that $\beta_{0}=b_{0}$ where $\beta_{0}, b_{0}$ denote the constant term in $\beta, b$ respectively. Similarly, by looking at the $e_{x}$ component in $\alpha \cdot f_{x}+\beta \cdot f_{y}+\gamma \cdot u^{r e} e_{y}$ and since $\left[p^{-1} k_{y}-k_{x}\right]+j e<s_{x}(p-1)$ we obtain $a_{0} \cdot g(\eta)=\beta_{0} \eta$. Hence, for all $g \in \operatorname{Gal}\left(K / \mathbf{Q}_{p}\right)$ there exists a unit $x_{g} \in\left(\mathbf{F}_{p^{f}} \otimes_{\mathbf{F}_{p}} \mathbf{F}\right)^{\times}$such that $g(\eta)=x_{g} \eta$. If $\eta \neq 0$ is not invertible in $\mathbf{F}_{p^{f}} \otimes_{\mathbf{F}_{p}} \mathbf{F}$, then there exist $g_{0} \in \operatorname{Gal}\left(K / \mathbf{Q}_{p}\right)$ and an idempotent element $e_{i} \in \mathbf{F}_{p^{f}} \otimes_{\mathbf{F}_{p}} \mathbf{F}$ such that $e_{i} \cdot \eta=0$ and $e_{i} \cdot g_{0}(\eta) \neq 0$. In particular one obtains $e_{i} \cdot g_{0}(\eta)=\left(e_{i} \cdot x_{g_{0}}\right)\left(e_{i} \cdot \eta\right)$ in $\mathbf{F}$, which is impossible as $e_{i} \cdot x_{g_{0}} \in \mathbf{F}^{\times}$. Thus $\eta \in\left(\mathbf{F}_{p^{f}} \otimes_{\mathbf{F}_{p}} \mathbf{F}\right)^{\times}$.

Since $s_{x}(p-1)-\left[p^{-1} k_{y}-k_{x}\right]_{f}-e j>0$ and $v_{0} \in u^{j e} \overline{\mathrm{S}}_{0}^{\times}$, there exists $P(u) \in \overline{\mathrm{S}}_{\mathbf{F}}$ such that

$$
f_{x}-P(u) f_{y}=\left[\begin{array}{c}
0 \\
u^{\left(s_{x}+s_{y}\right)(p-1)-\left[p^{-1} k_{y}-k_{x}\right]_{f}-e j} \epsilon_{1}
\end{array}\right]
$$

where $\epsilon_{1} \in \overline{\mathrm{S}}_{\mathbf{F}}^{\times}$, and so

$$
\operatorname{Fil}^{r} \mathcal{M}=\left\langle\left[\begin{array}{c}
0 \\
u^{\left(s_{x}+s_{y}\right)(p-1)-\left[p^{-1} k_{y}-k_{x}\right]_{f}-e j}
\end{array}\right], \quad\left[\begin{array}{c}
u^{\left[p^{-1} k_{y}-k_{x}\right]_{f}} v_{0} \\
u^{s_{y}(p-1)}
\end{array}\right]\right\rangle+u^{r e} \mathcal{M}
$$

Since $(r+j) e+\left[p^{-1} k_{y}-k_{x}\right]_{f}<\left(s_{x}+s_{y}\right)(p-1)$,

$$
\mathrm{Fil}^{r} \mathcal{M}=\left\langle\left[\begin{array}{c}
0 \\
u^{r e}
\end{array}\right], \quad\left[\begin{array}{c}
u^{\left[p^{-1} k_{y}-k_{x}\right]_{f}} v_{0} \\
u^{s_{y}(p-1)}
\end{array}\right]\right\rangle
$$

This implies $\mathrm{Fil}^{r} \mathcal{M} \otimes_{\overline{\mathrm{S}}_{\mathbf{F}}} \overline{\mathrm{S}}_{\mathbf{F}} /(u) \cong \omega_{f}^{k_{y}} \oplus \omega_{f}^{p^{-1} k_{y}}$, which is impossible as soon as $p^{-1} k_{x} \not \equiv k_{y}$. Therefore, $j>j_{0}$, in which case the sequence (4) splits, as we have seen before.

The following lemma is well-known and in most cases proved in [EGH13], proof of Theorem 3.3.13.

Lemma 3.3. Let $\rho: G_{\mathbf{Q}_{p}} \rightarrow \mathrm{GL}_{3}(E)$ be a potentially semi-stable representation with HodgeTate weights $\{-2,-1,0\}$ and with Galois type $\tau$.

Then

$$
\left.\operatorname{det}(\rho)\right|_{I_{\mathbf{Q}_{p}}}= \begin{cases}\varepsilon_{p}^{3} \cdot[\omega]^{x+y+z} & \text { if } \left.\tau \cong[\omega]^{x} \oplus[\omega]\right]^{y} \oplus[\omega]^{z} \\ \varepsilon_{p}^{3} \cdot[\omega]^{x+y} & \text { if } \tau \cong[\omega]^{x} \oplus\left[\omega_{2}\right]^{y} \oplus\left[\omega_{2}\right]^{p y} \\ \varepsilon_{p}^{3} \cdot[\omega]^{x} & \text { if } \tau \cong\left[\omega_{3}\right]^{x} \oplus\left[\omega_{3}\right]^{p x} \oplus\left[\omega_{3}\right]^{p^{2} x}\end{cases}
$$

where $\varepsilon_{p}$ is the cyclotomic character.

Proof. By hypothesis $\operatorname{det}(\rho)$ is a potentially crystalline character with parallel Hodge-Tate weights -3 and satisfies

according to the Galois type of $\rho$.

$$
\left.\mathrm{WD}(\operatorname{det}(\rho))\right|_{I_{\mathbf{Q}_{p}}}=\left\{\begin{array}{l}
{[\omega]^{x+y+z}} \\
{[\omega]^{x+y}} \\
{[\omega]^{x}}
\end{array}\right.
$$

In other words, each of $\operatorname{det}(\rho)[\omega]^{-(x+y+z)}, \operatorname{det}(\rho)[\omega]^{-(x+y)}, \operatorname{det}(\rho)[\omega]^{-x}$ is a crystalline character (with trivial Galois type) and with parallel Hodge-Tate weights -3 , i.e., it is isomorphic to $\varepsilon_{p}^{3}$ up to a twist by an unramified character. 


\section{Elimination of Galois types}

In this section we eliminate the Galois types for certain potentially crystalline lifts of an ordinary and strongly generic Galois representation $\bar{\rho}: G_{\mathbf{Q}_{p}} \rightarrow \mathrm{GL}_{3}(\mathbf{F})$ whose Loewy length is less than 3. We first classify such representations in the following subsection.

4.1. The local Galois side. Let $\bar{\rho}: G_{\mathbf{Q}_{p}} \rightarrow \mathrm{GL}_{3}(\mathbf{F})$ be a continuous Galois representation. If $\bar{\rho}$ is ordinary, then we may assume that $\left.\bar{\rho}\right|_{I_{\mathbf{Q}_{p}}}$ has the following form:

$$
\left.\bar{\rho}\right|_{I_{\mathbf{Q}_{p}}} \cong\left[\begin{array}{ccc}
\omega^{a_{2}+1} & *_{1} & *_{2} \\
0 & \omega^{a_{1}+1} & *_{3} \\
0 & 0 & \omega^{a_{0}+1}
\end{array}\right] .
$$

We say that an ordinary representation $\bar{\rho}$ is generic if $a_{1}-a_{0}>1, a_{2}-a_{1}>1, p-2>a_{2}-a_{0}$ in the matrix (8).

We assume that $\bar{\rho}$ is ordinary, generic, and minimally non-split (i.e. $\ell \ell(\bar{\rho})=2$ ). Since its Loewy length is 2 , we can distinguish five isomorphism classes for $\left.\bar{\rho}\right|_{I_{\mathbf{Q}_{p}}}$ :

$\circ$ Type $\left(\mathrm{T}_{2}\right)$ if

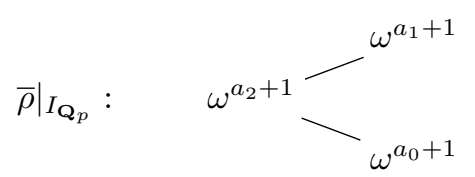

$\circ$ Type $\left(\mathrm{T}_{1}\right)$ if

$$
\begin{array}{lc} 
& \omega^{a_{2}+1}-\omega^{a_{1}+1} \\
\left.\bar{\rho}\right|_{\mathbf{Q}_{p}}: & \oplus \\
& \omega^{a_{0}+1}
\end{array}
$$

- Type $\left(\mathrm{T}_{0}\right)$ if

$$
\begin{array}{lc} 
& \omega^{a_{2}+1}-\omega^{a_{0}+1} \\
\left.\bar{\rho}\right|_{\mathbf{Q}_{p}}: & \oplus \\
& \omega^{a_{1}+1}
\end{array}
$$

○ Type $\left(\mathrm{T}_{-1}\right)$ if

$$
\begin{array}{lc} 
& \omega^{a_{1}+1}-\omega^{a_{0}+1} \\
\left.\bar{\rho}\right|_{\mathbf{Q}_{p}}: & \oplus \\
& \omega^{a_{2}+1}
\end{array}
$$

- Type $\left(\mathrm{T}_{-2}\right)$ if

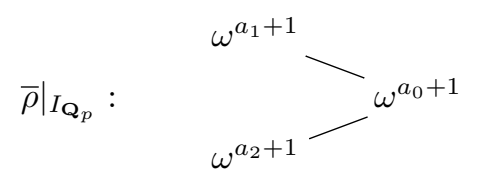

By $\omega^{a}-\omega^{b}$, we mean a non-trivial extension of $\omega^{b}$ by $\omega^{a}$. We also note that, for each $i \in\{0,1,2\}$, Type $\left(\mathrm{T}_{\mathrm{i}}\right)$ is dual to Type $\left(\mathrm{T}_{-\mathrm{i}}\right)$ up to twist.

We now introduce the following terminology:

Definition 4.1. An ordinary Galois representation $\bar{\rho}: G_{\mathbf{Q}_{p}} \rightarrow \mathrm{GL}_{3}(\mathbf{F})$ is said to be strongly generic if the integers $\left(a_{2}, a_{1}, a_{0}\right)$ in the matrix (8) satisfy the condition:

$$
a_{2}-a_{1}>2, a_{1}-a_{0}>2, p-3>a_{2}-a_{0} .
$$


In this paper we only consider an ordinary and strongly generic $\bar{\rho}$; in particular, such $\bar{\rho}$ is always Fontaine-Laffaille (cf. [HM], Proposition 2.1.4).

Let $\bar{\rho}$ be an ordinary and strongly generic representation of $G_{\mathbf{Q}_{p}}$, and let $\mathcal{M}$ be a rankthree Breuil module with descent data to $\mathbf{Q}_{p}$ of niveau $f$ such that $\mathrm{T}_{\mathrm{st}}^{*}(\mathcal{M}) \cong \bar{\rho}$. By Proposition 2.2.4 in $[\mathrm{HM}], \mathcal{M}$ is a successive extension of rank-one Breuil modules $\mathcal{M}_{i} \stackrel{\text { def }}{=}$ $\mathcal{M}\left(k_{i}, r_{i}, \lambda_{i}\right)$ such that

$$
\left.\omega_{f}^{k_{i}+p r_{i}} \cong \mathrm{T}_{\mathrm{st}}^{*}\left(\mathcal{M}_{i}\right)\right|_{I_{\mathbf{Q}_{p}}} \cong \omega^{a_{i}+1}
$$

for $i=0,1,2$ (the first isomorphism coming from Lemma 3.1). In this section, we consider only Type $\left(\mathrm{T}_{\mathrm{i}}\right)$ for $i=0,1,2$ and their semi-simpification $\bar{\rho}^{s s}$. Hence, $*_{3}=0$ in the matrix (8), and so $\mathcal{M}$ has two rank-two Breuil submodules (of weight 2), denoted by $\mathcal{M}_{2,1}$ and $\mathcal{M}_{2,0}$ and which correspond respectively to the (possibly split!) extensions $\omega^{a_{2}+1} \cdots \omega^{a_{1}+1}$ and $\omega^{a_{2}+1} \cdots \omega^{a_{0}+1}$ appearing as subrepresentations in $\bar{\rho}$. In particular, for $i \in\{0,1\}$ we have extensions

$$
0 \rightarrow \mathcal{M}_{2} \rightarrow \mathcal{M}_{2, i} \rightarrow \mathcal{M}_{i} \rightarrow 0
$$

If $i \in\{0,1\}$ and $\bar{\rho}$ is of Type ( $\left.\mathrm{T}_{\mathrm{i}}\right)$, then the exact sequence (11) is non-split for $i$; if $\bar{\rho}$ is of Type $\left(\mathrm{T}_{2}\right)$, then the exact sequences (11) are non-split for both $i=0$ and $i=1$. Finally, note that we have two exact sequences of Breuil modules

$$
0 \rightarrow \mathcal{M}_{2, i} \rightarrow \mathcal{M} \rightarrow \mathcal{M}_{j_{i}} \rightarrow 0
$$

where $j_{i}=1-i$ for $i=0,1$ (again by [HM], Proposition 2.2.4).

We keep these notations in the following subsections.

4.2. Galois types in niveau 1. In this subsection we let $f=1$ and $e=p-1$. In particular, all of the Breuil modules here are defined over $\mathbf{F}[u] / u^{e p}$. We set $K \stackrel{\text { def }}{=} \mathbf{Q}_{p}(\sqrt[e]{-p})$ and keep the notations as in Section 4.1.

Proposition 4.2. Let $\rho_{0}: G_{\mathbf{Q}_{p}} \rightarrow \mathrm{GL}_{3}(E)$ be a p-adic Galois representation, becoming crystalline over $K$ with Hodge-Tate weights $\{0,-1,-2\}$ and of Galois type $\left.\mathrm{WD}\left(\rho_{0}\right)\right|_{\mathbf{I}_{\mathbf{Q}_{p}}} \cong$ $[\omega]^{k_{2}} \oplus[\omega]^{k_{1}} \oplus[\omega]^{k_{0}}$.

If $\bar{\rho}_{0}^{s s} \cong \bar{\rho}^{s s}$, then the integers $k_{i} \in \mathbf{Z}$ satisfy $k_{i} \equiv a_{i}+1-r_{i} \bmod e$ with $r_{i} \in\{0,1,2\}$,

$$
r_{0}+r_{1}+r_{2}=3 \text {. }
$$

Moreover, if $\bar{\rho}_{0} \cong \bar{\rho}$, then the triple $\left(r_{2}, r_{1}, r_{0}\right)$ satisfies the following additional conditions: $r_{2} \neq 0$ and

○ if $\bar{\rho}$ is of Type $\left(\mathrm{T}_{2}\right)$, then $\left(r_{2}, r_{1}\right) \neq(1,2) \neq\left(r_{2}, r_{0}\right)$;

- if $\bar{\rho}$ is of Type $\left(\mathrm{T}_{1}\right)$, then $\left(r_{2}, r_{1}\right) \neq(1,2)$;

- if $\bar{\rho}$ is of Type $\left(\mathrm{T}_{0}\right)$, then $\left(r_{2}, r_{0}\right) \neq(1,2)$.

Proof. The first part of the proposition is [HM], Proposition 2.4.3. We recall its proof here, as it will be needed to use the splitting lemma.

We may assume that the rank-one Breuil modules $\mathcal{M}_{i}$ is of weight 2 . So $0 \leq r_{i} \leq 2$ for $i=0,1,2$. By the equation (10), we have $k_{i} \equiv a_{i}+1-r_{i} \bmod e$. By looking at the determinant of $\bar{\rho}$ we deduce, from Lemma 3.3, the conditions

$$
\omega^{3+k_{2}+k_{1}+k_{0}}=\left.\operatorname{det} \mathrm{T}_{\mathrm{st}}^{*}(\mathcal{M})\right|_{I_{\mathbf{Q}_{p}}}=\left.\operatorname{det} \bar{\rho}\right|_{I_{\mathbf{Q}_{p}}}=\omega^{a_{2}+a_{1}+a_{0}+3}
$$

and hence we have $r_{2}+r_{1}+r_{0}=3$. 
The main ingredient to complete the proof is now Lemma 3.2, specialized to $f=1$ and $r=2$. If $r_{2}=0$, then it is obvious that the exact sequence $0 \rightarrow \mathrm{Fil}^{2} \mathcal{M}_{2} \rightarrow \mathrm{Fil}^{2} \mathcal{M}_{2, i} \rightarrow$ $\mathrm{Fil}^{2} \mathcal{M}_{i} \rightarrow 0$ splits and so $\mathrm{T}_{\text {st }}^{*}\left(\mathcal{M}_{2, i}\right)$ splits. Hence, for any type of $\bar{\rho}$, we have $r_{2} \neq 0$. By Lemma 3.2 again, if $\left(r_{2}, r_{i}\right)=(1,2)$ then $\mathcal{M}_{2, i}$ splits as a direct sum of two characters. Hence, if $\bar{\rho}$ is of Type $\left(\mathrm{T}_{2}\right)$ we have $\left(r_{2}, r_{1}\right) \neq(1,2) \neq\left(r_{2}, r_{0}\right)$; if $\bar{\rho}$ is of Type $\left(\mathrm{T}_{0}\right)$ then $\left(r_{2}, r_{0}\right) \neq(1,2)$; if $\bar{\rho}$ is of Type $\left(\mathrm{T}_{1}\right)$ then $\left(r_{2}, r_{1}\right) \neq(1,2)$.

4.3. Galois types in niveau 2. In this subsection we let $f=2$ and $e=p^{2}-1$. In particular, all of the Breuil modules here are defined over $\mathbf{F}_{p^{2}} \otimes_{\mathbf{F}_{p}} \mathbf{F}[u] / u^{e p}$. We set $K \stackrel{\text { def }}{=}$ $\mathbf{Q}_{p^{2}}(\sqrt[e]{-p})$ and keep the notations as in Section 4.1.

The main result is the following:

Proposition 4.3. Let $\rho_{0}: G_{\mathbf{Q}_{p}} \rightarrow \mathrm{GL}_{3}(E)$ be a p-adic Galois representation, becoming crystalline over $K$ with Hodge-Tate weights $\{0,-1,-2\}$ and of Galois type $\left.\mathrm{WD}\left(\rho_{0}\right)\right|_{I_{\mathbf{Q}_{p}}} \cong$ $[\omega]^{x} \oplus\left[\omega_{2}\right]^{y} \oplus\left[\omega_{2}\right]^{p y}$.

If $\bar{\rho}_{0}^{s s} \cong \bar{\rho}^{s s}$, then the quintuple $\left(x, y, r_{2}, r_{1}, r_{0}\right)$ of integers satisfies one of the following possibilities:

(i) if $k_{2} \equiv(p+1) x \bmod e, k_{1} \equiv y \bmod e$, and $k_{0} \equiv p y \bmod e$, then

$$
\left\{\begin{array}{l}
x \equiv a_{2}-\delta \bmod (p-1) \text { and } y \equiv a_{1}+p a_{0}+\delta-\epsilon(p-1) \bmod e \\
r_{2}=(\delta+1)(p+1) \\
r_{1}=\left(a_{1}-a_{0}\right)+(2-\delta)-(\delta+\epsilon-1)(p-1) \\
r_{0}=(1+\epsilon)(p-1)-\left(a_{1}-a_{0}\right)+(2-\delta)
\end{array}\right.
$$

(ii) if $k_{2} \equiv p y \bmod e, k_{1} \equiv(p+1) x \bmod e$, and $k_{0} \equiv y \bmod e$, then

$$
\left\{\begin{array}{l}
x \equiv a_{1}+\delta \bmod (p-1) \text { and } y \equiv a_{0}+p a_{2}-\delta+\epsilon(p-1) \bmod e \\
r_{2}=(1-\epsilon)(p-1)-\left(a_{0}-a_{2}\right)+(2+\delta) \\
r_{1}=(1-\delta)(p+1) \\
r_{0}=\left(a_{0}-a_{2}\right)+(2+\delta)+(\delta+\epsilon+1)(p-1)
\end{array}\right.
$$

(iii) if $k_{2} \equiv y \bmod e, k_{1} \equiv p y \bmod e$, and $k_{0} \equiv(p+1) x \bmod e$, then

$$
\left\{\begin{array}{l}
x \equiv a_{0}-\delta \bmod (p-1) \text { and } y \equiv a_{2}+p a_{1}+\delta-\epsilon(p-1) \bmod e \\
r_{2}=\left(a_{2}-a_{1}\right)+(2-\delta)-(\delta+\epsilon-1)(p-1) \\
r_{1}=(1+\epsilon)(p-1)-\left(a_{2}-a_{1}\right)+(2-\delta) \\
r_{0}=(\delta+1)(p+1)
\end{array}\right.
$$

where $\epsilon \in\{0,1\}$ and $\delta \in\{-1,0,1\}$ satisfy $0 \leq \delta+\epsilon \leq 1$ and $y \not \equiv 0 \bmod (p+1)$.

Moreover, if $\bar{\rho}_{0} \cong \bar{\rho}$, then the pair $(\delta, \epsilon)$ satisfies the following additional conditions:

$\circ$ if $\bar{\rho}$ is of Type $\left(\mathrm{T}_{2}\right)$, then

$-\epsilon \neq 1$ in case $(\mathrm{i})$,

$-(\delta, \epsilon) \neq(-1,1)$ in case (ii),

$-\delta+\epsilon \neq 1$ in case (iii);

$\circ$ if $\bar{\rho}$ is of Type $\left(\mathrm{T}_{1}\right)$, then

$-(\delta, \epsilon) \neq(-1,1)$ in case $(\mathrm{i})$,

$-(\delta, \epsilon) \neq(-1,1)$ in case (ii);

- if $\bar{\rho}$ is of Type $\left(\mathrm{T}_{0}\right)$, then

$-\epsilon \neq 1$ in case (i),

$-\delta+\epsilon \neq 1$ in case (iii). 
Proof. The first part of the statement is $[\mathrm{HM}]$, Proposition 2.4.6: we recall its proof as this will be necessary to use the splitting lemma.

Let $\widehat{\mathcal{M}}$ be the strongly divisible module associated to a lattice in $\rho_{0}$ via Theorem 2.1 and write $\mathcal{M} \in \mathbf{F}$-BrMod ${ }_{\mathrm{dd}}^{2}$ for the associated Breuil module. Recall that $\mathcal{M}$ is endowed with a filtration by Breuil submodules (cf. the introduction to this section) whose graded pieces $\mathcal{M}_{i}=\mathcal{M}_{i}\left(k_{i}, r_{i}, \lambda_{i}\right)$ satisfy $\left.\mathrm{T}_{\mathrm{st}}^{*}\left(\mathcal{M}_{i}\right)\right|_{I_{\mathbf{Q}_{p}}} \cong \omega^{a_{i}+1}$ for $i=0,1,2$. In particular by Lemma 3.1 we have $0 \leq r_{i} \leq 2(p+1), k_{i}+p r_{i} \equiv 0 \bmod p+1$, and the equation (10). Let us write $\left.\mathrm{WD}\left(\rho_{0}\right)\right|_{I_{\mathbf{Q}_{p}}}=[\omega]^{x} \oplus\left[\omega_{2}\right]^{y} \oplus\left[\omega_{2}\right]^{p y}$ for the Galois type on $\rho_{0}$ (hence on $\mathcal{M}$ ). Therefore we may have either one of the following:

(i) $k_{2}=(p+1) x, k_{1}=y$, and $k_{0}=p y$;

(ii) $k_{2}=p y, k_{1}=(p+1) x$, and $k_{0}=y$;

(iii) $k_{2}=y, k_{1}=p y$, and $k_{0}=(p+1) x$.

Each of (i), (ii), (iii) above gives rise to the corresponding statement on $(x, y)$ in the same numbering of the proposition above. We consider only the case (i) (but all the other cases are very similar).

Assume the case (i). A direct manipulation from (10) provides us with

$$
(p+1) x+p r_{2} \equiv\left(a_{2}+1\right)(p+1) \quad \text { and } \quad p r_{1}-r_{0} \equiv\left(a_{1}-a_{0}\right)(p+1) \bmod e .
$$

In particular we obtain $r_{2} \equiv 0$ and $p r_{1}-r_{0} \equiv 0$ modulo $e$ and we may let $r_{2}=\delta^{\prime}(p+1)$ with $\delta^{\prime} \in\{0,1,2\}$ and $r_{1}+r_{0}=\alpha(p+1)$ for $\alpha \in\{1,2,3\}$ (due to the constraints $0 \leq r_{i} \leq 2(p+1)$ ). Thus, from (12), we now deduce $r_{1} \equiv a_{1}-a_{0}+\alpha \bmod (p-1)$.

Let us write $r_{1}=a_{1}-a_{0}+\alpha+\epsilon^{\prime}(p-1)$ so that $r_{0}=\alpha(p+1)-\left(a_{1}-a_{0}+\alpha\right)-\epsilon^{\prime}(p-1)$. As $y=a_{1}+p a_{0}+(p+1)-p \alpha+\epsilon^{\prime}(p-1)$ and $x \equiv a_{2}-1+\delta^{\prime} \bmod (p-1)$, we obtain, from Lemma 3.3,

$$
a_{2}+a_{1}+a_{0} \equiv x+y=a_{2}+1-\delta^{\prime}+a_{1}+p a_{0}+(p+1)-p \alpha+\epsilon^{\prime}(p-1) \bmod (p-1)
$$

hence the relation $\delta^{\prime}+\alpha=3$. By the constraints on the $r_{i}$ 's we see that if $\alpha=1$ then $\epsilon^{\prime}=0$, if $\alpha=2$ then $\epsilon^{\prime} \in\{0,1\}$ and if $\alpha=3$ then $\epsilon^{\prime}=1$. We may describe the pair $\left(\delta^{\prime}, \epsilon^{\prime}\right)$ as

$$
\delta^{\prime} \in\{0,1,2\} \text { and } \epsilon^{\prime} \in\{0,1\} \text { with } 1 \leq \delta^{\prime}+\epsilon^{\prime} \leq 2 .
$$

and by letting $\delta=\delta^{\prime}-1$ and $\epsilon=2-\left(\delta^{\prime}+\epsilon^{\prime}\right)$ we obtain finally case (i).

Note that we have shown that

$$
(\mathrm{i}) \Rightarrow\left\{\begin{array}{l}
r_{2}=(\delta+1)(p+1) ; \\
r_{1}=\left(a_{1}-a_{0}\right)+(2-\delta)+(\delta+\epsilon-1)(p-1) ; \\
r_{0}=(1+\epsilon)(p-1)-\left(a_{1}-a_{0}\right)+(2-\delta) .
\end{array}\right.
$$

In a completely analogous fashion cases (ii) and (iii) provide us with:

$$
\text { (ii) } \Rightarrow\left\{\begin{array}{l}
r_{2}=(1-\epsilon)(p-1)-\left(a_{0}-a_{2}\right)+(2+\delta) ; \\
r_{1}=(1-\delta)(p+1) ; \\
r_{0}=\left(a_{0}-a_{2}\right)+(2+\delta)+(\delta+\epsilon+1)(p-1),
\end{array}\right.
$$

and

$$
\text { (iii) } \Rightarrow\left\{\begin{array}{l}
r_{2}=\left(a_{2}-a_{1}\right)+(2-\delta)+(\delta+\epsilon-1)(p-1) ; \\
r_{1}=(1+\epsilon)(p-1)-\left(a_{2}-a_{1}\right)+(2-\delta) ; \\
r_{0}=(\delta+1)(p+1) .
\end{array}\right.
$$

We can now apply Lemma 3.2 to the Breuil submodules $\mathcal{M}_{2,0}, \mathcal{M}_{2,1}$ in $\mathcal{M}$.

For $\mathcal{M}_{2,0}$, one can readily check that $r_{2}(p-1) \leq\left[p^{-1} k_{0}-k_{2}\right]_{2}$ when $(\delta, \epsilon)=(-1,1)$ in the case (i) or when $\delta+\epsilon=1$ in case (iii). Similarly, for $\mathcal{M}_{2,1}, r_{2}(p-1) \leq\left[p^{-1} k_{1}-k_{2}\right]_{2}$ when $(\delta, \epsilon)=(-1,1)$ in case (i) or $(\delta, \epsilon)=(-1,1)$ in case (ii). Hence, for these cases, $\mathrm{Fil}^{2} \mathcal{M}_{2, j}$ 
splits and so does $\mathrm{T}_{\mathrm{st}}^{*}\left(\mathcal{M}_{2, j}\right)$ by Lemma 3.2. Moreover, when $(\delta, \epsilon)=(0,1)$ in case (i), $\mathrm{T}_{\mathrm{st}}^{*}\left(\mathcal{M}_{2,0}\right)$ splits as well: indeed, in the notations of Lemma 3.2 , we now have $j_{0}=0$, and moreover $\left[p^{-1} k_{0}-k_{2}\right]_{2}=e-[(p+1) x-y],\left(r_{2}+r_{0}\right)(p-1)-\left[p^{-1} k_{0}-k_{2}\right]_{2}>2 e$; hence $\mathrm{T}_{\mathrm{st}}^{*}\left(\mathcal{M}_{2,0}\right)$ splits again.

4.4. Galois types in niveau 3. In this subsection we let $f=3$ and $e=p^{3}-1$. In particular, all of the Breuil modules here are defined over $\mathbf{F}_{p^{3}} \otimes_{\mathbf{F}_{p}} \mathbf{F}[u] / u^{e p}$. We set $K \stackrel{\text { def }}{=}$ $\mathbf{Q}_{p^{3}}(\sqrt[e]{-p})$ and keep the notations as in Section 4.1.

The main result is the following:

Proposition 4.4. Let $\rho_{0}: G_{\mathbf{Q}_{p}} \rightarrow \mathrm{GL}_{3}(E)$ be a p-adic Galois representation, becoming crystalline over $K$ with Hodge-Tate weights $\{0,-1,-2\}$ and of Galois type $\left.\mathrm{WD}\left(\rho_{0}\right)\right|_{\mathbf{I}_{\mathbf{Q}_{p}}} \cong$ $\left[\omega_{3}\right]^{x} \oplus\left[\omega_{3}\right]^{p x} \oplus\left[\omega_{3}\right]^{p^{2} x}$.

If $\bar{\rho}_{0}^{s s} \cong \bar{\rho}^{s s}$, then the quadruple $\left(x, r_{2}, r_{1}, r_{0}\right)$ of integers satisfies the following properties:

$$
x=\left(\delta_{2}+a_{2}\right)+\left(\delta_{1}+a_{1}\right)+\left(\delta_{0}+a_{0}\right)
$$

and

(i) if $k_{2} \equiv x \bmod e, k_{1} \equiv p x \bmod e$, and $k_{0} \equiv p^{2} x \bmod e$, then

$$
\left\{\begin{array}{l}
r_{2}=\left(p^{2}+p+1\right)+(p+1)\left(\delta_{2}+a_{2}\right)-p^{2}\left(\delta_{1}+a_{1}\right)-p^{2}\left(\delta_{0}+a_{0}\right) \\
r_{1}=\left(p^{2}+p+1\right)-\left(\delta_{2}+a_{2}\right)+\left(p^{2}+p\right)\left(\delta_{1}+a_{1}\right)-\left(\delta_{0}+a_{0}\right) \\
r_{0}=\left(p^{2}+p+1\right)-p\left(\delta_{2}+a_{2}\right)-p\left(\delta_{1}+a_{1}\right)+\left(p^{2}+1\right)\left(\delta_{0}+a_{0}\right)
\end{array}\right.
$$

(ii) if $k_{2} \equiv p^{2} x \bmod e, k_{1} \equiv p x \bmod e$, and $k_{0} \equiv x \bmod e$, then

$$
\left\{\begin{array}{l}
r_{2}=\left(p^{2}+p+1\right)+\left(p^{2}+1\right)\left(\delta_{2}+a_{2}\right)-p\left(\delta_{1}+a_{1}\right)-p\left(\delta_{0}+a_{0}\right) \\
r_{1}=\left(p^{2}+p+1\right)-\left(\delta_{2}+a_{2}\right)+\left(p^{2}+p\right)\left(\delta_{1}+a_{1}\right)-\left(\delta_{0}+a_{0}\right) \\
r_{0}=\left(p^{2}+p+1\right)-p^{2}\left(\delta_{2}+a_{2}\right)-p^{2}\left(\delta_{1}+a_{1}\right)+(p+1)\left(\delta_{0}+a_{0}\right),
\end{array}\right.
$$

for $\delta_{i} \in(p-1) \mathbf{Z}$ such that $0 \leq r_{i} \leq 2\left(p^{2}+p+1\right)$ for all $i$ and $x \not \equiv 0 \bmod \left(p^{2}+p+1\right)$.

Proof. We only give a complete proof of the case (i). The same argument works for (ii) (Or just swap $r_{2}$ and $r_{0}$ from the case (i)). By equation (10), we have for each $i$ :

$$
p^{2-i} x+p r_{i} \equiv\left(a_{i}+1\right)\left(p^{2}+p+1\right) \bmod e .
$$

By Lemma 3.3 , we have $\omega^{x+3} \cong \operatorname{det} \mathrm{T}_{\mathrm{st}}^{*}(\mathcal{M}) \cong \omega^{a_{2}+a_{1}+a_{0}+3}$ and so

$$
x \equiv a_{2}+a_{1}+a_{0} \bmod (p-1) .
$$

We let $x=a_{2}+a_{1}+a_{0}+\epsilon(p-1)$. Adding all the equations in (13) we get:

$$
x\left(1+p+p^{2}\right)+p\left(r_{2}+r_{1}+r_{0}\right) \equiv\left(a_{2}+a_{1}+a_{0}+3\right)\left(p^{2}+p+1\right) \bmod e .
$$

Since $x \equiv a_{2}+a_{1}+a_{0} \bmod (p-1)$, we have

$$
r_{2}+r_{1}+r_{0}=3\left(p^{2}+p+1\right) .
$$

From the equation (13), we may let

$$
\left\{\begin{array}{l}
r_{2}=\left(a_{2}+1\right)\left(p^{2}+p+1\right)-p^{2}\left(a_{2}+a_{1}+a_{0}+\epsilon(p-1)\right)+\delta_{2}^{\prime}\left(p^{3}-1\right) \\
r_{1}=\left(a_{1}+1\right)\left(p^{2}+p+1\right)-\left(a_{2}+a_{1}+a_{0}+\epsilon(p-1)\right)+\delta_{1}^{\prime}\left(p^{3}-1\right) \\
r_{0}=\left(a_{0}+1\right)\left(p^{2}+p+1\right)-p\left(a_{2}+a_{1}+a_{0}+\epsilon(p-1)\right)+\delta_{0}^{\prime}\left(p^{3}-1\right) .
\end{array}\right.
$$

By equation (14), we have the relation $\epsilon=\delta_{2}^{\prime}+\delta_{1}^{\prime}+\delta_{0}^{\prime}$. Letting $\delta_{i}=\delta_{i}^{\prime}(p-1)$, this completes the proof of the case (i). 


\section{Local/Global compatibility and Serre Weights}

Let $F$ be a CM field in which $p$ splits completely. In this section we prove our main results on modular weights for a Galois representation $\bar{r}: G_{F} \rightarrow \mathrm{GL}_{3}(\mathbf{F})$ which is ordinary at all places $w \mid p$ (Theorems 5.9 and 5.10). After some reminders on the space of algebraic automorphic forms in Section 5.1, having [EGH13], Section 7 as a main reference, we prove the key statement on inertial local/global compatibility in Section 5.2 (Theorem 5.5). The latter theorem enables us to use the computations in Section 4 to obtain one of the main results on modular weights (Theorem 5.9). We also prove that the obvious weights in the predicted set is modular (Theorem 5.10).

In this section $v$ and $v^{\prime}$ (resp. $w$ and $w^{\prime}$ ) are always places of $F^{+}$(resp. of $F$ ).

5.1. The space of automorphic forms on certain unitary groups. Let $F / \mathbf{Q}$ be a CM field, $F^{+}$its maximal totally real subfield. For the rest of the paper, we assume that $p$ splits completely in $F$. (We note that the assumption that $p$ splits completely in $F$ is unnecessary in this subsection and next, although we may need that every place in $F^{+}$dividing $p$ splits in $F$. However, for brevity we assume that $p$ splits completely in $F$, which is the context for our main results, Theorems 5.9 and 5.10.)

We let $G_{/ F^{+}}$be a reductive group, which is an outer form for $\mathrm{GL}_{3}$, and which splits over $F$. We assume that $G\left(F_{v}^{+}\right) \simeq U_{3}(\mathbf{R})$ for all $v \mid \infty$. We recall ([EGH13], Section 7.1) that $G$ admits a reductive model $\mathcal{G}$ defined over $\mathcal{O}_{F^{+}}\left[N^{-1}\right]$ for an integer $N$ that is prime to $p$ together with an isomorphism

$$
\iota: \mathcal{G}_{/ \mathcal{O}_{F}\left[N^{-1}\right]} \stackrel{\sim}{\rightarrow} \mathrm{GL}_{3 / \mathcal{O}_{F}\left[N^{-1}\right]}
$$

Let $W$ be an $\mathcal{O}_{E^{-}}$-module endowed with a smooth action of $\mathcal{G}\left(\mathcal{O}_{F^{+}, p}\right)$ and let $U \leq$ $G\left(\mathbf{A}_{F^{+}}^{\infty, p}\right) \times \mathcal{G}\left(\mathcal{O}_{F^{+}, p}\right)$ be a compact open subgroup. The space of algebraic automorphic forms on $G$ of level $U$ and coefficients in $W$ is defined as the following $\mathcal{O}_{E}$-module:

$$
S(U, W) \stackrel{\text { def }}{=}\left\{f: G\left(F^{+}\right) \backslash G\left(\mathbf{A}_{F^{+}}^{\infty}\right) \rightarrow W \mid f(g u)=u_{p}^{-1} f(g) \forall g \in G\left(\mathbf{A}_{F^{+}}^{\infty}\right), u \in U\right\}
$$

(with the obvious notation $u=u^{p} u_{p}$ for the elements in $U$ ).

We recall that the level $U$ is said to be sufficiently small if there exists a finite place $v$ in $F^{+}$such that the image of the projection $U \rightarrow G\left(F_{v}^{+}\right)$does not contain elements of finite order except the identity. For a finite place $v$ of $F^{+}$we say that $U$ is unramified at $v$ if one has a decomposition $U=\mathcal{G}\left(\mathcal{O}_{F_{v}^{+}}\right) U^{v}$ for some compact open $U^{v} \leq G\left(\mathbf{A}_{F+}^{\infty, v}\right)$. If $w$ is a finite place of $F$ we say, with an abuse, that $w$ is an unramified place for $U$ if its restriction $\left.w\right|_{F^{+}}$ is unramified for $U$.

Let $\mathcal{P}_{U}$ denote the set consisting of finite places $w$ of $F$ such that $\left.v \stackrel{\text { def }}{=} w\right|_{F^{+}}$is split in $F$, $w \nmid p N$ and $U$ is unramified at $w$. If $\mathcal{P} \subseteq \mathcal{P}_{U}$ is a subset of finite complement we write $\mathbf{T}_{\mathcal{P}}=\mathcal{O}_{E}\left[T_{w}^{(i)}, w \in \mathcal{P}, i \in\{1,2,3\}\right]$ for the universal Hecke algebra on $\mathcal{P}$, where the Hecke operator $T_{w}^{(i)}$ acts on the space $S(U, W)$ as the usual double coset operator

$$
\left[\mathrm{GL}_{3}\left(\mathcal{O}_{F_{w}}\right)\left(\begin{array}{cc}
\varpi_{w} \mathrm{Id}_{i} & 0 \\
0 & \mathrm{Id}_{3-i}
\end{array}\right) \mathrm{GL}_{3}\left(\mathcal{O}_{F_{w}}\right)\right]
$$

via the evident isomorphism

$$
\iota_{w}: \mathcal{G}\left(\mathcal{O}_{F_{v}^{+}}\right) \stackrel{\sim}{\rightarrow} \mathcal{G}\left(\mathcal{O}_{F_{w}}\right) \stackrel{\iota}{\rightarrow} \mathrm{GL}_{3}\left(\mathcal{O}_{F_{w}}\right) .
$$

We briefly recall the relation between the space $\mathcal{A}$ of classical automorphic forms and the previous spaces of algebraic automorphic forms, in the particular case which is relevant to us. 
Following [EGH13], Section 7.3 we consider the subset $\bigoplus_{w \mid p}\left(\mathbf{Z}_{+}^{3}\right)_{0}$ of dominant weights $\underline{\lambda}=\left(\underline{\lambda}_{w}\right)_{w}$ verifying the condition

$$
\lambda_{1, w^{c}}+\lambda_{3, w}=0, \quad \lambda_{2, w}+\lambda_{2, w^{c}}=0, \quad \lambda_{3, w^{c}}+\lambda_{1, w}=0
$$

for all triples $\underline{\lambda}_{w}=\left(\lambda_{1, w}, \lambda_{2, w}, \lambda_{3, w}\right)$ and all pairs $\left(w, w^{c}\right)$. We write $W_{\underline{\lambda}_{w}}$ for the $\mathcal{O}_{F_{w}}{ }^{-}$ specialization of the dual Weyl module associated to $\underline{\lambda}_{w}$ (cf. [EGH13], Section 4.1.1); by condition (17) one deduces an isomorphism of $\mathcal{G}\left(\mathcal{O}_{F_{v}^{+}}\right)$-representations $W_{\underline{\lambda}_{w}} \circ \iota_{w} \cong W_{\underline{\lambda}_{w^{c}}} \circ \iota_{w^{c}}$. Therefore, by letting $W_{\underline{\lambda}_{v}} \stackrel{\text { def }}{=} W_{\underline{\lambda}_{w}} \circ \iota_{w}$ for any place $w \mid v$, the $\mathcal{G}\left(\mathcal{O}_{F^{+}, p}\right)$-representation

$$
W_{\underline{\lambda}} \stackrel{\text { def }}{=} \bigotimes_{v \mid p} W_{\underline{\lambda}_{v}}
$$

is well defined.

For a weight $\underline{\lambda} \in \bigoplus_{w \mid p}\left(\mathbf{Z}_{+}^{3}\right)_{0}$ let us write $S_{\underline{\lambda}}\left(\overline{\mathbf{Q}}_{p}\right)$ to denote the inductive limit of the spaces $S\left(U, W_{\underline{\lambda}} \otimes_{\mathcal{O}_{E}} \overline{\mathbf{Q}}_{p}\right)$ over the compact open subgroups $U \leq G\left(\mathbf{A}_{F^{+}}^{\infty}\right) \times \mathcal{G}\left(\mathcal{O}_{F^{+}, p}\right)$ (note that the latter is an inductive system in a natural way, with injective transition maps induced from the inclusions between levels). Then $S_{\underline{\underline{\lambda}}}\left(\overline{\mathbf{Q}}_{p}\right)$ has a natural smooth left action of $G\left(\mathbf{A}_{F^{+}}^{\infty}\right)$ induced from the right translation of functions.

Fix an isomorphism $\imath: \overline{\mathbf{Q}}_{p} \stackrel{\sim}{\rightarrow} \mathbf{C}$. As we have done for the $\mathcal{O}_{F_{w}}$-specialization of the dual Weyl modules, we can define a smooth $G\left(F_{\infty}^{+}\right)$-representation with $\mathbf{C}$-coefficients $\sigma_{\lambda} \cong$ $\bigoplus \sigma_{\underline{\lambda}_{v}}$, where $\sigma_{\underline{\lambda}_{v}}$ depends only on $\underline{\lambda}_{w}$ for a place $w \mid v$ (we invite the reader to refer to $v \mid \infty$

[EGH13], Section 7.1.4 for the precise definition of $\sigma_{\underline{\lambda}}$ ).

Lemma 5.1. The isomorphism $\imath: \overline{\mathbf{Q}}_{p} \stackrel{\sim}{\rightarrow} \mathbf{C}$ induces an isomorphism of smooth $G\left(\mathbf{A}_{F^{+}}^{\infty}\right)$ representations

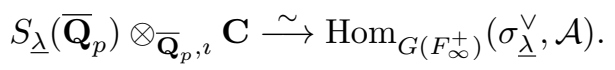

5.2. Potentially crystalline lifts and Serre weights. The aim of this subsection is to prove the inertial local/global compatibility for Hecke eigenclasses of certain automorphic forms attached to Galois representations $\bar{r}: G_{F} \rightarrow \mathrm{GL}_{3}(\mathbf{F})$, which is the key to obtain the upper bound in the set of modular weights. After some preliminaries on the inertial local Langlands correspondence we state the main result in Theorem 5.5; as this theorem is well known to experts, we decided to mainly refer to [EGH13], avoiding a self-contained exposition.

We start by recalling some facts about the tame inertial local Langlands correspondence via Deligne-Lusztig representations. We refer the reader to [Her09], Section 4 for a precise and concise reference.

Let $n \in\{1,2,3\}$ and define the extension $k_{w, n} / k_{w}$ by letting $\left[k_{w, n}: k_{w}\right]=n$. Let $T$ be a maximal torus in $\mathrm{GL}_{3 / k_{w}}$, stable under the Frobenius morphism on $\mathrm{GL}_{3 / k_{w}}$. Up to $\mathrm{GL}_{3 / k_{w}}$-conjugacy we have an identification

$$
T\left(k_{w}\right) \stackrel{\sim}{\longrightarrow} \prod_{j} k_{w, n_{j}}^{\times}
$$

where $3 \geq n_{j}>0$ and $\sum_{j} n_{j}=3$ (cf. [Her09], Lemma 4.7).

If $\theta: T\left(k_{w}\right) \rightarrow \overline{\mathbf{Q}}_{p}^{\times}$is a primitive character, i.e. $\theta_{j}: k_{w, n_{j}}^{\times} \rightarrow \overline{\mathbf{Q}}_{p}^{\times}$is primitive for all $j$ via the identification (18), then we have a well defined Deligne-Lusztig representation $R_{T}^{\theta}$. 
By letting $\Theta\left(\theta_{j}\right)$ be the cuspidal representation of $\mathrm{GL}_{n_{j}}\left(k_{w}\right)$ associated to the primitive character $\theta_{j}$ via [Her09], Lemma 4.4, we have

$$
R_{T}^{\theta} \cong \operatorname{Ind}_{P_{\underline{\underline{n}}}}^{\mathrm{GL}_{3}\left(k_{w}\right)} \otimes_{j} \Theta\left(\theta_{j}\right)
$$

where $P_{\underline{n}}$ is the standard parabolic subgroup containing the Levi $\prod_{j} \mathrm{GL}_{n_{j}}$.

Let $F_{w, n}$ be the unramified extension over $F_{w}$ of degree $n$; we consider $\theta_{j}$ as a character on $\mathcal{O}_{F_{w, n_{j}}}^{\times}$by inflation and we define the inertial character $\operatorname{rec}(\theta)$ as follows:

(i) $\operatorname{rec}(\theta)=\bigoplus_{j=1}^{3} \theta_{j} \circ \operatorname{Art}_{F_{w}}^{-1}$ if $\theta_{j}: k_{w} \rightarrow \mathbf{Q}_{p}^{\times}$are niveau one characters;

(ii) $\operatorname{rec}(\theta)=\theta_{1} \circ \operatorname{Art}_{F_{w}}^{-1} \oplus \bigoplus_{\sigma \in \operatorname{Gal}\left(k_{w, 2} / k_{w}\right)} \sigma\left(\theta_{2} \circ \operatorname{Art}_{F_{w, 2}}^{-1}\right)$ if $\theta_{1}$ is a niveau one character and $\theta_{2}$ is a niveau 2, primitive character on $k_{w, 2}^{\times}$;

(iii) $\operatorname{rec}(\theta)=\bigoplus_{\sigma \in \operatorname{Gal}\left(k_{w, 3} / k_{w}\right)} \sigma\left(\theta_{1} \circ \operatorname{Art}_{F_{w, 3}}^{-1}\right)$ if $\theta_{1}$ is a niveau three, primitive character on $k_{w, 3}$.

Proposition 5.2. Let $\Pi_{w}$ be an irreducible smooth $\mathrm{GL}_{3}\left(F_{w}\right)$-representation. Assume that $\left.R_{T}^{\theta} \hookrightarrow \Pi_{w}\right|_{\mathrm{GL}_{3}\left(\mathcal{O}_{F_{w}}\right)}$. Then

$$
\left.\operatorname{rec}_{F_{w}}\left(\Pi_{w}\right)\right|_{I_{F_{w}}} \cong \operatorname{rec}(\theta)
$$

and the monodromy operator on $\operatorname{rec}_{F_{w}}\left(\Pi_{w}\right)$ is zero.

Proof. If either $T\left(k_{w}\right) \cong k_{w, 3}^{\times}$or if $T$ splits over $k_{w}$ the result holds by [EGH13], Proposition 2.4.1.

Assume therefore that $T\left(k_{w}\right) \cong k_{w, 2}^{\times} \times k_{w}^{\times}$and write $\theta=\theta_{2} \otimes \theta_{1}$ where $\theta_{i}: k_{w, i} \rightarrow E^{\times}$.

In this case, we deduce from [Her09], Lemma 6.7 that $R_{T}^{\theta} \cong \operatorname{ind}_{P\left(k_{w}\right)}^{\mathrm{GL}_{3}\left(k_{w}\right)}\left(R_{T_{2}}^{\theta_{2}} \otimes \theta_{1}\right)$ where $T_{2}$ is a maximal torus of $\mathrm{GL}_{2}\left(k_{w}\right)$ such that $T_{2}\left(k_{w}\right) \cong k_{w, 2}^{\times}$and $P_{/ \mathcal{O}_{F}}$ denotes the standard parabolic (with the usual Levi decomposition $P=M \cdot N$ ) defined by $P_{1}(A)=\left[\begin{array}{lll}* & * & * \\ * & * & * \\ 0 & 0 & *\end{array}\right]$ for any $\mathcal{O}_{F_{w}}$-algebra $A$.

Let us write $\sigma \stackrel{\text { def }}{=} R_{T_{2}}^{\theta_{2}} \otimes \theta_{1}$, which will be identified with a representation of $M\left(\mathcal{O}_{F_{w}}\right)$ by inflation from $M\left(k_{w}\right)$, and $\sigma_{\mathfrak{P}}$ for the inflation of $\sigma$ to the standard parahoric $\mathfrak{P} \subseteq \mathrm{GL}_{3}\left(\mathcal{O}_{F_{w}}\right)$ associated to $P\left(k_{w}\right)$.

By [Mor99] Lemma 3.6, we have an isomorphism

$$
\pi^{\sigma \mathfrak{P}} \stackrel{\sim}{\longrightarrow}\left(\pi_{N}\right)^{\sigma}
$$

$\pi_{N}$ is the Jacquet module and the superscripts denote the isotypical components (with respect to the evident Hecke algebras acting on $\pi$ and $\pi_{N}$ ).

As $\pi^{\sigma_{\mathfrak{P}}} \neq 0$ by assumption (and by Frobenius reciprocity) we deduce an $M\left(\mathcal{O}_{F_{w}}\right)$ equivariant morphism $\sigma \hookrightarrow \pi_{N}$. As the pair $\left(M\left(\mathcal{O}_{F_{w}}\right), \sigma\right)$ is a $M\left(F_{w}\right)$-type, we deduce an $M\left(F_{w}\right)$-equivariant morphism $\pi_{N} \rightarrow \tau_{\sigma}$ where $\tau_{\sigma}=\tau_{2} \otimes \tau_{1}$ is supercuspidal, and

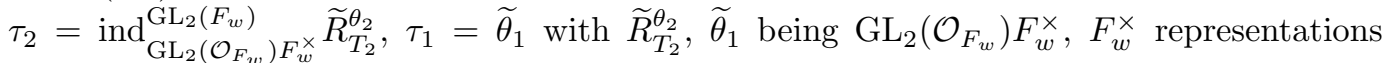
extending $R_{T_{2}}^{\theta_{2}}, \theta_{1}$ respectively (this is [Mor99], Proposition 4.1)

Normalizing the Jacquet functor via the (unramified) modulus character $\delta_{P}$, Frobenius reciprocity finally provides us with a non-zero map $\pi \rightarrow \imath_{P\left(F_{w}\right)}^{\mathrm{GL}_{3}\left(F_{w}\right)} \tau_{\sigma}$ which is moreover an isomorphism (the $\mathrm{GL}_{3}\left(F_{w}\right)$-representations here being irreducible).

By the classical local Langlands correspondence ([HT01], [Hen00]) we deduce the isomorphism $\operatorname{rec}_{F_{w}}\left(\imath_{P\left(F_{w}\right)}^{\mathrm{GL}_{3}\left(F_{w}\right)} \tau_{\sigma}\right) \cong \operatorname{rec}_{F_{w}}\left(\tau_{2}\right) \oplus \operatorname{rec}_{F_{w}}\left(\tau_{1}\right)$ up to an unramified twist, and the 
result follows from the inertial local Langlands correspondence for $\mathrm{GL}_{2}\left(F_{w}\right) \times G L_{1}\left(F_{w}\right)$ (cf. Henniart's appendix to [BM02]).

Definition 5.3. A Serre weight for $\mathcal{G}$ (or just Serre weight if $\mathcal{G}$ is clear from the context) is an isomorphism class of a smooth, absolutely irreducible representation $V$ of $\mathcal{G}\left(\mathcal{O}_{F^{+}, p}\right)$.

As explained in [EGH13], Section 7.3, a Serre weight $V$ admits an explicit description in terms of the $\mathrm{GL}_{3}\left(k_{w}\right)$-representations $F(x, y, z)$. More precisely, let $w$ be a place of $F$ above $p$ and write $\left.v \stackrel{\text { def }}{=} w\right|_{F^{+}}$. For any restricted triple $\underline{a}_{w} \stackrel{\text { def }}{=}\left(a_{w}, b_{w}, c_{w}\right) \in \mathbf{Z}_{+}^{3}$ we consider the Serre weight $F_{\underline{a}_{w}}=F\left(a_{w}, b_{w}, c_{w}\right)$. It is an irreducible representation of $\mathrm{GL}_{3}\left(k_{w}\right)$, hence of $\mathcal{G}\left(k_{v}\right)$ via the isomorphism $\iota_{w}$. Note that $F\left(a_{w}, b_{w}, c_{w}\right)^{\vee} \circ \iota_{w^{c}} \cong F\left(a_{w}, b_{w}, c_{w}\right) \circ \iota_{w}$ as $\mathcal{G}\left(k_{v}\right)$-representations, i.e. $F\left(a_{w^{c}}, b_{w^{c}}, c_{w^{c}}\right) \circ \iota_{w^{c}} \cong F\left(a_{w}, b_{w}, c_{w}\right) \circ \iota_{w}$ as soon as

$$
a_{w^{c}}+c_{w}=0, \quad b_{w}+b_{w^{c}}=0 \quad \text { and } \quad c_{w^{c}}+a_{w}=0 .
$$

In particular, if $\underline{a} \in \bigoplus_{w \mid p}\left(\mathbf{Z}_{+}^{3}\right)_{0}$ is restricted, the smooth $\mathcal{G}\left(\mathcal{O}_{F_{v}^{+}}\right)$-representation $F_{\underline{a}_{v}} \stackrel{\text { def }}{=}$ $F_{\underline{a}_{w}} \circ \iota_{w}$ is well defined and we set

$$
F_{\underline{a}} \stackrel{\text { def }}{=} \bigotimes_{v \mid p} F_{\underline{a}_{v}}
$$

which is a Serre weight for $\mathcal{G}\left(\mathcal{O}_{F^{+}, p}\right)$. (One may notice that our description of Serre weights is different from that in [EGH13], but of course it will give the same notion under the assumption that $p$ splits completely in $F$.)

From [EGH13], Lemma 7.3.4 if $V$ is a Serre weight for $\mathcal{G}$, one has $V \circ \iota_{w}^{-1} \cong F_{a}$ for some $\underline{a} \in \bigoplus_{w \mid p}\left(\mathbf{Z}_{+}^{3}\right)_{0}$.

Definition 5.4. Let $\bar{r}: G_{F} \rightarrow \mathrm{GL}_{3}(\mathbf{F})$ be a continuous Galois representation and let $V$ be a Serre weight for $\mathcal{G}$. We say that $\bar{r}$ is modular of weight $V$ (or that $V$ is a modular weight for $\bar{r})$ if there exists a compact open subset $U$ in $G\left(\mathbf{A}_{F}^{\infty, p}\right) \times \mathcal{G}\left(\mathcal{O}_{F^{+}, p}\right)$ and a cofinite subset $\mathcal{P}$ in the set of finite places of $F$, lying over split places of $F^{+}$not dividing $p N$ and unramified for both $\bar{r}$ and $U$, such that

$$
S(U, V)_{\overline{\mathfrak{m}}} \neq 0
$$

where $\overline{\mathfrak{m}}$ is the kernel of the system of Hecke eigenvalues $\bar{\alpha}: \mathbf{T}_{\mathcal{P}} \rightarrow \mathbf{F}$ associated to $\bar{r}$, i.e.

$$
\operatorname{det}\left(1-\bar{r}^{\vee}\left(\operatorname{Frob}_{w}\right) X\right)=\sum_{j=0}^{3}(-1)^{j}\left(\mathbf{N}_{F_{w} / \mathbf{Q}_{p}}(w)\right)^{\left(\begin{array}{l}
j \\
2
\end{array}\right)} \bar{\alpha}\left(T_{w}^{(j)}\right) X^{j}
$$

for all $w \in \mathcal{P}$.

We write

$$
W_{\bmod }(\bar{r})
$$

for the set of all modular weights for $\bar{r}$.

The following theorem is the main result of this subsection, being the key to prove weight elimination for $\bar{r}$. As it is well known to experts we decided to mainly refer to [EGH13], thus avoiding a self contained exposition.

Theorem 5.5. Let $V=F_{\underline{a}}$ be a modular weight for the Galois representation $\bar{r}: G_{F} \rightarrow$ $\mathrm{GL}_{3}(\mathbf{F})$. Fix a place $w \mid p$ and assume that $F_{\underline{a}_{v}} \circ \iota_{w}^{-1}$ is a Jordan-Hölder constituent in the mod-p reduction of a Deligne-Lusztig representation $R_{T}^{\theta}$ of $\mathrm{GL}_{3}\left(k_{w}\right)$, where $T_{/ k_{w}}$ is a Frobenius stable maximal torus in $\mathrm{GL}_{3}\left(k_{w}\right)$ and $\theta: T\left(k_{w}\right) \rightarrow \overline{\mathbf{Q}}_{p}^{\times}$is a primitive regular character. 
Then $\left.\bar{r}\right|_{G_{F_{w}}}$ has a potentially crystalline lift with parallel Hodge-Tate weights $\{-2,-1,0\}$ and Galois type $\operatorname{rec}(\theta)$.

Before the proof, we recall the following results.

Lemma 5.6. Assume that $U$ is sufficiently small and let $M$ be a smooth $\mathcal{O}_{E}\left[\mathcal{G}\left(\mathcal{O}_{F^{+}, p}\right)\right]$ module of finite type over $\mathcal{O}_{E}$. Let $\mathbf{T}$ be an anemic Hecke algebra acting on $S(U, M)$, as defined in Section 5.1 and $\bar{\alpha}: \mathbf{T} \rightarrow \mathbf{F}$ be a system of Hecke eigenvalues such that $S\left(U, M \otimes_{\mathcal{O}_{E}} \mathbf{F}\right)_{\operatorname{ker}(\bar{\alpha})} \neq 0$.

Then, up to enlarging $\mathcal{O}_{E}$, there exists a system of Hecke eigenvalues $\alpha: \mathbf{T} \rightarrow \mathcal{O}_{E}$ such that $S(U, M)_{\operatorname{ker}(\alpha)} \neq 0$ and $\alpha \equiv \bar{\alpha} \bmod \varpi$.

Theorem 5.7. Let $\Pi \subseteq S_{\underline{\lambda}}\left(\overline{\mathbf{Q}}_{p}\right)$ be an irreducible $G\left(\mathbf{A}_{F^{+}}^{\infty}\right)$-representation. Fix an isomorphism $\iota: \overline{\mathbf{Q}}_{p} \rightarrow \mathbf{C}$ and let $|\cdot|^{\frac{1-n}{2}}$ be the unique square root of $|\cdot|^{(1-n)}$ taking positive values when composed with $\iota$.

Then there exists a continuous semisimple representation

$$
r_{\Pi}: G_{F} \rightarrow \mathrm{GL}_{3}\left(\overline{\mathbf{Q}}_{p}\right)
$$

such that

(i) $r_{\Pi}^{c} \otimes \varepsilon^{n-1} \cong r_{\Pi}^{\vee}$.

(ii) For each place $w$ above $p$, the representation $\left.r_{\Pi}\right|_{G_{F w}}$ is de Rham with Hodge-Tate weights

$$
\operatorname{HT}\left(\left.r_{\Pi}\right|_{G_{F}}\right)=\left\{\lambda_{w, 1}+2, \lambda_{w, 2}+1, \lambda_{w, 3}\right\} .
$$

(iii) If $v \nmid p$ is a finite place of $F^{+}$which splits as $v=w w^{c}$ in $F$, then

$$
\mathrm{WD}\left(\left.r_{\Pi}\right|_{G_{F_{w}}}\right)^{\mathrm{F}-\mathrm{ss}} \cong \operatorname{rec}_{w}\left(\left(\Pi_{v} \circ \iota_{w}^{-1}\right) \otimes|\cdot|^{(1-n) / 2}\right)
$$

(where we recall the isomorphism $G\left(F_{v}^{+}\right) \rightarrow \mathrm{GL}_{3}\left(F_{w}\right)$ ).

(iv) If $w \mid p$ is a place of $F$ and $\left.v \stackrel{\text { def }}{=} w\right|_{F^{+}}$, then

$$
\mathrm{WD}\left(\left.r_{\Pi}\right|_{G_{F}}\right)^{\mathrm{F}-\mathrm{ss}} \cong \operatorname{rec}_{w}\left(\left(\Pi_{v} \circ \iota_{w}^{-1}\right) \otimes|\cdot|^{(1-n) / 2}\right) .
$$

Proof. Cf. [EGH13], proof of Theorem 7.2.1. We remark that the assertion (iv) is the main result of $[\mathrm{Car}]$.

Proof of Theorem 5.5. The proof follows verbatim the argument of the "proof of Axiom Ã3" in [EGH13], Proposition 7.4.4.

First of all, note that "Axiom Â1" holds true in our situation (it does not require any condition on $\left.\left.\bar{r}\right|_{G_{F_{w}}}\right)$. Let $\left.v \stackrel{\text { def }}{=} w\right|_{F^{+}}$and $\underline{\lambda} \in \bigoplus_{w^{\prime} \mid p}\left(\mathbf{Z}_{+}^{3}\right)_{0}$ be the restricted weight defined by $\underline{\lambda}_{w^{\prime}}=\underline{a}_{w^{\prime}}$ if $\left.w^{\prime}\right|_{F^{+}} \neq v$ and $\underline{\lambda}_{w^{\prime}}=0$ if $\left.w^{\prime}\right|_{F^{+}}=v$.

Let us write $R \stackrel{\text { def }}{=} R_{T}^{\theta}$ for the Deligne-Lusztig representation of $\mathrm{GL}_{3}\left(k_{w}\right)$, which will be identified with a $\mathcal{G}\left(k_{v}\right)$-representation via $\iota_{w}$ (and hence with a $\mathcal{G}\left(\mathcal{O}_{F_{v}^{+}}\right.$)-representation by inflation). By the argument in the "proof of Axiom Â" in [EGH13], Proposition 7.4.4 we obtain

$$
\operatorname{Hom}_{\mathcal{G}\left(\mathcal{O}_{F_{v}}\right)}\left(R^{\vee}, S\left(U^{v} K_{v}(1), W_{\underline{\lambda}} \otimes_{\overline{\mathbf{Z}}_{p}} \overline{\mathbf{Q}}_{p}\right)_{\mathfrak{m}}\right) \neq 0
$$

where $\mathfrak{m}$ is the kernel of a system of Hecke eigenvalues $\alpha: \mathbf{T} \rightarrow \mathcal{O}_{E}$ lifting $\bar{\alpha}$ as in the statement of Lemma 5.6 and $K_{v}(1)$ is the kernel of the morphism $\mathcal{G}\left(\mathcal{O}_{F_{v}^{+}}\right) \stackrel{\iota_{\dddot{w}}}{\rightarrow} \mathrm{GL}_{3}\left(\mathcal{O}_{F_{w}}\right) \rightarrow$ $\mathcal{G}\left(k_{w}\right)$.

Hence there exists an automorphic irreducible representation $\Pi \subseteq S_{\lambda}\left(\overline{\mathbf{Q}}_{p}\right)$ such that $\Pi^{U^{v} K_{v}(1)} \cap S\left(U^{v} K_{v}(1), \overline{\mathbf{Q}}_{p}\right)_{\mathfrak{m}} \neq 0$ and $\Pi_{v}$ contains $R^{\vee}$. 
Let $r_{\Pi}: G_{F} \rightarrow \mathrm{GL}_{3}\left(\overline{\mathbf{Q}}_{p}\right)$ be as in the statement of Theorem 5.7. By Chebotarev density theorem, Theorem 5.7-(iii) and [CHT08], Corollary 3.1.2 we have $\bar{r}_{\Pi} \cong \bar{r}^{\vee}$. Moreover, we have that $\left.r_{\Pi}^{\vee}\right|_{G_{F_{w}}}$ is potentially crystalline, with Hodge-Tate weights $\{-2,-1,0\}$ and Galois type $\operatorname{rec}(\theta)$, by Theorem 5.7-(iv), (ii) and Proposition 5.2.

It is therefore the required lift.

5.3. Modular weights and main results. In this subsection we prove our main results on Serre weights for a modular Galois representation $\bar{r}: G_{F} \rightarrow \mathrm{GL}_{3}(\mathbf{F})$ which is ordinary at places above $p$. After recalling the definition of the set of modular weights associated to $\bar{r}$ we define, for each place $w \mid p$ in $F$, a set of conjectural weights $W_{w}^{?}(\bar{r})$ which depends on the shape of the submodule structure of $\left.\bar{r}\right|_{G_{F_{w}}}$. We often write $W_{w}^{?}\left(\left.\bar{r}\right|_{G_{F_{w}}}\right)$ for $W_{w}^{?}(\bar{r})$.

Let $w \mid p$ be a place of $F$. We assume that $\left.\bar{r}\right|_{G_{F_{w}}}$ is ordinary and strongly generic. (Remember that we assume $p$ splits completely in $F$.) According to the isomorphism class of $\left.\bar{r}\right|_{G_{F_{w}}}$ (cf. Section 4.1), we define the following set of Serre weights:

$$
W_{w}^{?}(\bar{r}) \stackrel{\text { def }}{=}\left\{F\left(a_{2}-1, a_{1}, a_{0}+1\right), F\left(a_{0}+(p-1), a_{1}, a_{2}-(p-1)\right)\right\} \cup \mathcal{W}
$$

where

(i) if $\left.\bar{r}\right|_{G_{F_{w}}}$ is of Type $\left(\mathrm{T}_{2}\right)$

$$
\mathcal{W} \stackrel{\text { def }}{=}\left\{F\left(a_{2}-1, a_{0},\left(a_{1}+1\right)-(p-1)\right)\right\} ;
$$

(ii) if $\left.\bar{r}\right|_{G_{F_{w}}}$ is of Type $\left(\mathrm{T}_{1}\right)$

$$
\mathcal{W} \stackrel{\text { def }}{=}\left\{\begin{array}{cc}
F\left(a_{2}-1, a_{0}, a_{1}+1-(p-1)\right), & F\left(a_{0}-1+(p-1), a_{2}, a_{1}+1\right) \\
F\left(a_{1}+(p-1), a_{2}, a_{0}\right), & F\left(a_{2}, a_{0}, a_{1}-(p-1)\right)
\end{array}\right\} ;
$$

(iii) if $\left.\bar{r}\right|_{G_{F_{w}}}$ is of Type $\left(\mathrm{T}_{0}\right)$

$$
\mathcal{W} \stackrel{\text { def }}{=}\left\{F\left(a_{2}-1, a_{0},\left(a_{1}+1\right)-(p-1)\right), \quad F\left(a_{1}-1+(p-1), a_{2}, a_{0}+1\right)\right\} ;
$$

(iv) if $\left.\bar{r}\right|_{G_{F}}$ is of Type $\left(\mathrm{T}_{-1}\right)$

$$
\mathcal{W} \stackrel{\text { def }}{=}\left\{\begin{array}{cc}
F\left(a_{1}-1+(p-1), a_{2}, a_{0}+1\right), & F\left(a_{1}-1, a_{0}, a_{2}+1-(p-1)\right) \\
F\left(a_{1}+(p-1), a_{2}, a_{0}\right), & F\left(a_{2}, a_{0}, a_{1}-(p-1)\right)
\end{array}\right\}
$$

(v) if $\left.\bar{r}\right|_{G_{F_{w}}}$ is of Type $\left(\mathrm{T}_{-2}\right)$

$$
\mathcal{W} \stackrel{\text { def }}{=}\left\{F\left(a_{1}-1+(p-1), a_{2}, a_{0}+1\right)\right\} .
$$

Recall that if $\left.\bar{r}\right|_{G_{F}}$ is maximally non-split, i.e. $\ell \ell\left(\left.\bar{r}\right|_{G_{F_{w}}}\right)=3$, then one can define a Fontaine-Laffaille parameter $\mathrm{FL}\left(\left.\bar{r}\right|_{G_{F_{w}}}\right) \in \mathbf{P}^{1}(\mathbf{F})$ (cf. [HM], Section 2). In this case, we set $\mathcal{W} \stackrel{\text { def }}{=} \emptyset$ provided that $\mathrm{FL}\left(\left.\bar{r}\right|_{G_{F_{w}}}\right) \in \mathbf{P}^{1}(\mathbf{F}) \backslash\{0, \infty\}$.

If $\left.\bar{r}\right|_{G_{F w}} \simeq \omega^{a_{2}+1} \oplus \omega^{a_{1}+1} \oplus \omega^{a_{0}+1}$, then we define

$$
W_{w}^{?}(\bar{r}) \stackrel{\text { def }}{=} W_{L} \cup W_{U} \cup W_{S}
$$

where

$$
\begin{gathered}
W_{L} \stackrel{\text { def }}{=}\left\{\begin{array}{c}
F\left(a_{2}-1, a_{1}, a_{0}+1\right), F\left(a_{1}-1, a_{0}, a_{2}+1-(p-1)\right) \\
F\left(a_{0}-1+(p-1), a_{2}, a_{1}+1\right)
\end{array}\right\} ; \\
W_{U} \stackrel{\text { def }}{=}\left\{\begin{array}{c}
F\left(a_{0}-1+(p-1), a_{1}, a_{2}+1-(p-1)\right), F\left(a_{2}-1, a_{0}, a_{1}+1-(p-1)\right) \\
F\left(a_{1}-1+(p-1), a_{2}, a_{0}+1\right)
\end{array}\right\} ; \\
W_{S} \stackrel{\text { def }}{=}\left\{\begin{array}{c}
F\left(a_{0}+(p-1), a_{1}, a_{2}-(p-1), F\left(a_{2}, a_{0}, a_{1}-(p-1)\right)\right. \\
F\left(a_{1}+(p-1), a_{2}, a_{0}\right)
\end{array}\right\} .
\end{gathered}
$$


Note that $W_{w}^{?}\left(\left.\bar{r}\right|_{G_{F_{w}}}\right) \subset W_{w}^{?}\left(\left.\bar{r}\right|_{G_{F_{w}}} ^{s s}\right)$ for each type of $\left.\bar{r}\right|_{G_{F_{w}}}$. The weights in $W_{L}$ and in $W_{U}$ are called obvious weights and those in $W_{S}$ shadow weights. We let

$$
W_{w}^{?, o b v}(\bar{r}) \stackrel{\text { def }}{=} W_{w}^{?}(\bar{r}) \cap\left(W_{L} \cup W_{U}\right)
$$

for each isomorphism class of $\left.\bar{r}\right|_{G_{F_{w}}}$.

As in [EGH13], Section 7.5, we define the set of predicted modular weighs for $\bar{r}$ in terms of the sets $W_{w}^{?}(\bar{r})$ : if $v \mid p$ is a place of $F^{+}, w \mid v$ is a place of $F$, and $V_{v}$ is an absolutely irreducible $\mathcal{G}\left(k_{v}\right)$-representation over $\mathbf{F}$, we introduce the conditions

$$
V_{v} \circ \iota_{w}^{-1} \in W_{w}^{?}(\bar{r})
$$

and

$$
V_{v} \circ \iota_{w}^{-1} \in W_{w}^{?, o b v}(\bar{r}) .
$$

Following Remark 7.5.2 in [EGH13] we see that $V_{v} \circ \iota_{w}^{-1} \in W_{w}^{?}(\bar{r})$ is equivalent to $V_{v} \circ \iota_{w^{c}}^{-1} \in$ $W_{w^{c}}^{?}(\bar{r})$. We can therefore define the set of predicted Serre weights $W^{?}(\bar{r})$ as follows:

Definition 5.8. Assume that for all places $w \mid p$ the Galois representation $\left.\bar{r}\right|_{G_{F_{w}}}$ is ordinary, strongly generic (cf. Definition 4.1), and $\ell \ell\left(\left.\bar{r}\right|_{G_{F_{w}}}\right)<3$.

The set of predicted Serre weights for $\bar{r}$ is

$$
W^{?}(\bar{r}) \stackrel{\text { def }}{=}\left\{V=\bigotimes_{v \mid p} V_{v} \text { s.t. } V_{v} \text { satisfies condition }(21) \text { for all } v \mid p\right\} .
$$

We similarly define the set of predicted obvious weights for $\bar{r}$ to be

$$
W^{?, o b v}(\bar{r}) \stackrel{\text { def }}{=}\left\{V=\bigotimes_{v \mid p} V_{v} \text { s.t. } V_{v} \text { satisfies condition }(22) \text { for all } v \mid p\right\} \text {. }
$$

The main result to obtain the upper bound on the modular weights for $\bar{r}$ is the following:

Theorem 5.9. Let $F$ be an imaginary $C M$ field, and suppose that $p$ splits completely in $F$. Let $\bar{r}: G_{F} \rightarrow \mathrm{GL}_{3}(\mathbf{F})$ be a modular Galois representation. We assume that for all places $w \mid p$ the Galois representation $\left.\bar{r}\right|_{G_{F_{w}}}$ are ordinary and strongly generic. If moreover $\ell \ell\left(\left.\bar{r}\right|_{G_{F_{w}}}\right)=$ 3, we further assume that its Fontaine-Laffaille parameter satisfies $\operatorname{FL}\left(\left.\bar{r}\right|_{G_{F_{w}}}\right) \in \mathbf{P}^{1}(\mathbf{F}) \backslash$ $\{0, \infty\}$.

Then one has

$$
W_{\text {mod }}(\bar{r}) \subseteq W^{?}(\bar{r}) .
$$

The proof of Theorem 5.9 in the case $\ell \ell\left(\left.\bar{r}\right|_{G_{F_{w}}}\right)<3$ will be performed in the following subsection. The case that $\left.\bar{r}\right|_{G_{F_{w}}}$ is maximally non-split (i.e., $\ell \ell\left(\left.\bar{r}\right|_{G_{F_{w}}}\right)=3$ ) is one of the main results in $[\mathrm{HM}]$.

For $\bar{r}: G_{F} \rightarrow \mathrm{GL}_{3}(\mathbf{F})$ as in Theorem 5.9 , one expects the upper bound given by $W^{?}(\bar{r})$ to be the best possible. In general it is hard to decide whether a weight is actually modular for such a $\bar{r}$, but thanks to the results of [BLGG], the modularity of the obvious weights is now a corollary to Theorem 5.9.

In the following theorem, concerning the modularity of the obvious weights $W_{w}^{?, o b v}(\bar{r})$ (cf. (20)), we use the terminology of [BLGG]. We sincerely thank Daniel Le for pointing out the reference to [BLGGT], Lemma 1.4.3, which is crucial in the proof of Theorem 5.10. 
Theorem 5.10. Let $F$ be an imaginary $C M$ field with maximal totally real subfield $F^{+}$and suppose that $F / F^{+}$is unramified at all finite places and that $p$ splits completely in $F$. Let $\bar{r}: G_{F} \rightarrow \mathrm{GL}_{3}(\mathbf{F})$ be an irreducible representation with split ramification. Assume that there is a RACSDC automorphic representation $\Pi$ of $\mathrm{GL}_{3}\left(\mathbf{A}_{F}\right)$ of weight $\mu \in\left(\mathbf{Z}_{+}^{3}\right)_{0}^{\operatorname{Hom}(F, \mathbf{C})}$ and level prime to $p$ such that

$$
\begin{aligned}
& \circ \bar{r} \simeq \bar{r}_{p, i}(\Pi) \text {; } \\
& \circ \text { For each place } w \mid p \text { of } F,\left.r_{p, i}(\Pi)\right|_{G_{F_{w}}} \text { is potentially diagonalizable; } \\
& \circ \bar{r}\left(G_{F\left(\zeta_{p}\right)}\right) \text { is adequate. }
\end{aligned}
$$

Assume further that for all places $w \mid p$ the Galois representations $\left.\bar{r}\right|_{G_{F_{w}}}$ are ordinary, strongly generic, and of Loewy length $\ell \ell\left(\left.\bar{r}\right|_{G_{F_{w}}}\right)<3$.

Then one has

$$
W^{?, o b v}(\bar{r}) \subseteq W_{\text {mod }}(\bar{r}) \subseteq W^{?}(\bar{r}) .
$$

Proof. Let $V=\bigotimes_{v \mid p} V_{v}$ be a Serre weight with $V_{v} \circ \iota_{w}^{-1} \in W_{w}^{?, o b v}(\bar{r})$ for all $w \mid p$ and let $\underline{a} \in \bigoplus_{w \mid p}\left(\mathbf{Z}_{+}^{3}\right)_{0}$ correspond to $V$. By Theorem 4.1.9 and Lemma 5.1.1 in [BLGG] together with Theorem 5.9, it is enough to show that, for each $w \mid p$, the local representation $\left.\bar{r}\right|_{G_{F_{w}}}$ has a potentially diagonalizable crystalline lift with Hodge type $\underline{a}_{w}$. (Note that we don't have to consider a lift of $\underline{a}$ since $p$ splits completely in $F$.) But, by Lemma 1.4.3 in [BLGGT], it is enough to show that there exist an ordinary crystalline lift with Hodge type $\underline{a}_{w}$.

Let $m, n$ be integers with $m \geq n+2$ and $\chi_{m}$ (resp. $\chi_{n}$ ) be a $m$-th (resp. $n$-th) Tate twist of a unramified character. Then an extension of $\chi_{n}$ by $\chi_{m}$ is (ordinary) crystalline by [PR94], Proposition 3.1. The generic condition on $m, n \bmod (p-1)$ (i.e., $m \not \equiv n$ or $m \not \equiv n+1$ $\bmod (p-1))$ implies that the natural map $\operatorname{Ext}_{\mathbf{Z}_{p}\left[G_{\left.\mathbf{Q}_{p}\right]}\right]}^{1}\left(\chi_{n}, \chi_{m}\right) \rightarrow \operatorname{Ext}_{\mathbf{F}_{p}\left[G_{\mathbf{Q}_{p}}\right]}^{1}\left(\bar{\chi}_{n}, \bar{\chi}_{m}\right)$ is surjective. Hence, the cases for $\left.\bar{r}\right|_{G_{F_{w}}}$ of Type $\left(\mathrm{T}_{1}\right)$ and $\left(\mathrm{T}_{0}\right)$ and for $\left.\left.\bar{r}\right|_{G_{F_{w}}} \simeq \bar{r}\right|_{G_{F_{w}}} ^{s s}$ hold immediately from this.

The only non-trivial case is for $\left.\bar{\rho} \stackrel{\text { def }}{=} \bar{r}\right|_{G_{F_{w}}}$ of Type $\left(\mathrm{T}_{2}\right)$. Let $\left.\bar{\rho}\right|_{I_{\mathbf{Q}_{p}}} ^{s s}=\omega^{a_{2}+1} \oplus \omega^{a_{1}+1} \oplus \omega^{a_{0}+1}$ with the strongly generic condition on $a_{i}$. We may consider $\bar{\rho}$ as a quotient of $\bar{\rho}_{1} \oplus \bar{\rho}_{2}$ by $\bar{\rho}_{0}$ where $\bar{\rho}_{1}$ and $\bar{\rho}_{2}$ are the two 2-dimensional subrepresentations of $\bar{\rho}$ and $\bar{\rho}_{0}$ is the 1dimensional subrepresentation of $\bar{\rho}$. Let $\underline{a}_{w}=\left(a_{w}, b_{w}, c_{w}\right)$. (Then $\left\{a_{w}+2, b_{w}+1, c_{w}\right\} \equiv$ $\left\{a_{2}+1, a_{1}+1, a_{0}+1\right\} \bmod (p-1)$ since $V$ is an obvious weight.) By the argument in the previous paragraph, $\bar{\rho}_{1}$ and $\bar{\rho}_{2}$ have ordinary crystalline lifts $\rho_{1}$ with Hodge-Tate weights $\left(a_{w}+2, b_{w}+1\right)$ and $\rho_{2}$ with Hodge-Tate weights $\left(a_{w}+2, c_{w}\right)$ respectively, both of which has a common subrepresentation $\rho_{0}$ that is a lift of $\bar{\rho}_{0}$ with Hodge-Tate weight $a_{w}+2$. Let $\rho$ be the quotient of $\rho_{1} \oplus \rho_{2}$ by $\rho_{0}$ (via the diagonal embedding). Then $\rho$ is obviously an ordinary crystalline 3 -dimensional representation with Hodge-Tate weights $\left(a_{w}+2, b_{w}+1, c_{w}\right)$ that is isomorphic to a lift of $\bar{\rho}$.

5.4. Proof of Theorem 5.9. We consider the situation in the statement of Theorem 5.9. Namely, we fix a place $w$ above $p$, we let $V=\bigotimes_{v^{\prime} \mid p} V_{v^{\prime}}$ be a modular weight for $\bar{r}$, and we assume that $\left.\bar{r}\right|_{G_{F_{w}}}$ is ordinary and strongly generic as in Definition 4.1.

We write $\left.\bar{\rho} \stackrel{\text { def }}{=} \bar{r}\right|_{G_{F_{w}}}$ and note that we can assume, without loss of generality, that $\bar{\rho}$ is either of Type $\left(\mathrm{T}_{\mathrm{i}}\right)$ for $i=0,1,2$. The other types are dual of those up to twist, and one has

$$
W_{\text {mod }}\left(\bar{r}^{\vee} \otimes \omega^{1-3}\right)=W_{\text {mod }}(\bar{r})^{\vee} .
$$

In all what follows, we write $F \stackrel{\text { def }}{=} V_{v} \circ \iota_{w}^{-1}$ where $\left.v \stackrel{\text { def }}{=} w\right|_{F^{+}}$. 
Recall that we write $R_{T}^{\theta}$ for a Deligne-Lusztig representation associated to a maximal torus $T_{/ k_{w}}$ and a primitive regular character $\theta: T\left(k_{w}\right) \rightarrow \overline{\mathbf{Q}}_{p}^{\times}$. We also recall that if $F \in \mathrm{JH}\left(\overline{R_{T}^{\theta}}\right)$, then, by Theorem $5.5,\left.\bar{r}\right|_{G_{F_{w}}}$ has a potentially crystalline lift with HodgeTate weights $\{-2,-1,0\}$ and with Galois type $\operatorname{rec}(\theta)$.

5.4.1. Niveau 1 types. We keep the assumption and the notation as at the beginning of Section 5.4

Proposition 5.11. If $V=\bigotimes_{v^{\prime} \mid p} V_{v^{\prime}}$ is a modular weight for $\bar{r}$, then $F=V_{v} \circ \iota_{w}^{-1}$ is isomorphic to one of the weights in the following list

$$
\begin{aligned}
& F\left(a_{2}, a_{0}, a_{1}-(p-1)\right), \quad F\left(a_{1}+(p-1), a_{2}, a_{0}\right), \quad F\left(a_{0}+(p-1), a_{1}, a_{2}-(p-1)\right), \\
& F\left(a_{2}-1, a_{0}+1, a_{1}-(p-1)\right), \quad F\left(a_{1}+(p-1), a_{2}-1, a_{0}+1\right), \quad F\left(a_{0}+p, a_{1}, a_{2}-p\right), \\
& F\left(a_{2}-1, a_{0}, a_{1}-p\right), \quad F\left(a_{1}+p, a_{2}-1, a_{0}\right), \quad F\left(a_{0}+(p-1), a_{1}+1, a_{2}-p\right), \\
& F\left(a_{1}, a_{0}, a_{2}-(p-1)\right), \quad F\left(a_{2}-1, a_{1}, a_{0}+1\right), \quad F\left(a_{0}+(p-1), a_{2}-1, a_{1}+1\right)
\end{aligned}
$$

with moreover the further weights:

$$
\begin{aligned}
& F\left(a_{2}+(p-1), a_{0}-1, a_{1}+1\right), \quad F\left(a_{1}+p, a_{2}, a_{0}-1\right), \quad F\left(a_{0}-1, a_{2}, a_{1}+p\right) \\
& F\left(a_{0}-1+(p-1), a_{1}+1, a_{2}-(p-1)\right)
\end{aligned}
$$

if $\bar{\rho}$ is of Type $\left(\mathrm{T}_{1}\right)$, and the further weights:

$$
\begin{aligned}
& F\left(p+a_{0}, a_{1}-1, a_{2}\right), \quad F\left(p-1+a_{2}, p+a_{0}, a_{1}-1\right), \quad F\left(p-2+a_{1}, a_{2}, a_{0}+1\right), \\
& F\left(p-2+a_{1}, p+a_{0}, a_{2}\right), \quad F\left(p-1+a_{0}, a_{2}, a_{1}\right)
\end{aligned}
$$

if $\bar{\rho}$ is of Type $\left(\mathrm{T}_{0}\right)$.

Proof. Let $T\left(k_{w}\right)$ be the maximal split torus of diagonal matrices, and let us write $F=$ $F\left(b_{2}, b_{1}, b_{0}\right)$ for a restricted weight $\left(b_{2}, b_{1}, b_{0}\right) \in X_{1}(T)$ (where $X_{1}(T)$ denotes the set of restricted weights as in [Her09], Definition 3.6).

By Frobenius reciprocity we have

$$
F \cong \operatorname{cosoc}\left(\operatorname{Ind}_{B\left(\mathbf{F}_{p}\right)}^{\mathrm{GL}_{3}\left(\mathbf{F}_{p}\right)} \omega^{b_{2}} \otimes \omega^{b_{1}} \otimes \omega^{b_{0}}\right)
$$

In particular, we have $F \in \mathrm{JH}\left(\overline{R_{T}^{\theta}}\right)$ where $\theta \cong[\omega]^{b_{2}} \otimes[\omega]^{b_{1}} \otimes[\omega]^{b_{0}}$ and hence by Theorem 5.5 we deduce that $\left.\bar{r}\right|_{G_{\mathbf{Q}_{p}}}$ admits a potentially crystalline lift $\rho: G_{\mathbf{Q}_{p}} \rightarrow \mathrm{GL}_{3}(E)$ whose associated Galois type has niveau one: $\left.\mathrm{WD}(\rho)\right|_{I_{\mathbf{Q}_{p}}} \cong[\omega]^{b_{2}} \oplus[\omega]^{b_{1}} \oplus[\omega]^{b_{0}}$.

By Proposition 4.2 specialized to Type $\left(\mathrm{T}_{2}\right)$ we may assume that

$$
b_{i}=a_{i}+1-r_{i}
$$

where the triple $\left(r_{2}, r_{1}, r_{0}\right)$ is as in Proposition 4.2 for Type $\left(\mathrm{T}_{2}\right)$.

Since the Serre weight $F\left(b_{2}, b_{1}, b_{0}\right)$ is an obvious constituent of the Jordan-Hölder factors of the principal series $\operatorname{Ind}_{B\left(\mathbf{F}_{p}\right)}^{\mathrm{GL}_{3}\left(\mathbf{F}_{p}\right)} \omega^{b_{2}} \otimes \omega^{b_{1}} \otimes \omega^{b_{0}}$ we deduce from $(24)$ that $F\left(b_{2}, b_{1}, b_{0}\right)$ is one of the 6 obvious weights in the semisimplification of one of the following principal series:

$$
\begin{gathered}
\operatorname{Ind}_{B\left(\mathbf{F}_{p}\right)}^{\mathrm{GL}_{3}\left(\mathbf{F}_{p}\right)} \omega^{a_{2}} \otimes \omega^{a_{1}} \otimes \omega^{a_{0}}, \quad \operatorname{Ind}_{B\left(\mathbf{F}_{p}\right)}^{\mathrm{GL}_{3}\left(\mathbf{F}_{p}\right)} \omega^{a_{2}-1} \otimes \omega^{a_{1}} \otimes \omega^{a_{0}+1} \\
\operatorname{Ind}_{B\left(\mathbf{F}_{p}\right)}^{\mathrm{GL}_{3}\left(\mathbf{F}_{p}\right)} \omega^{a_{2}-1} \otimes \omega^{a_{1}+1} \otimes \omega^{a_{0}} .
\end{gathered}
$$

Let us consider the following obvious weights in $\operatorname{Ind}_{B\left(\mathbf{F}_{p}\right)}^{\mathrm{GL}_{3}\left(\mathbf{F}_{p}\right)} \omega^{a_{2}} \otimes \omega^{a_{1}} \otimes \omega^{a_{0}}$ lying in the lower alcove:

$$
F_{1} \stackrel{\text { def }}{=} F\left(a_{0}+p-1, a_{2}, a_{1}\right), \quad F_{2} \stackrel{\text { def }}{=} F\left(a_{2}, a_{1}, a_{0}\right) .
$$


Each of them forms the cosocle of the following dual Weyl modules

$$
W\left(a_{1}+(p-2), a_{2}, a_{0}+1\right), W\left(p-2+a_{0}, a_{1}, a_{2}-(p-2)\right)
$$

and hence $F_{i} \in \mathrm{JH}\left(\overline{R_{T}^{\theta_{i}}}\right)$ where

$$
\theta_{1}=[\omega]^{a_{2}} \otimes[\omega]^{a_{1}-1} \otimes[\omega]^{a_{0}+1}, \theta_{2}=[\omega]^{a_{2}+1} \otimes[\omega]^{a_{1}} \otimes[\omega]^{a_{0}-1} .
$$

If $F_{1}$ (resp. $F_{2}$ ) were modular, by Theorem 5.5 we shall have a potentially crystalline lift of $\bar{\rho}$, with associated Galois type $\operatorname{rec}\left(\theta_{1}\right)$ (resp. $\left.\operatorname{rec}\left(\theta_{2}\right)\right)$ contradicting Proposition 4.2 for Type $\left(\mathrm{T}_{2}\right)$.

In a completely analogous fashion we see that weights

$$
F_{3} \stackrel{\text { def }}{=} F\left(a_{1}, a_{0}+1, a_{2}-1-(p-1)\right) \text { and } F_{4} \stackrel{\text { def }}{=} F\left(a_{0}+1+(p-1), a_{2}-1, a_{1}-(p-1)\right)
$$

(obvious weights of $\operatorname{Ind}_{B\left(\mathbf{F}_{p}\right)}^{\mathrm{GL}_{3}\left(\mathbf{F}_{p}\right)} \omega^{a_{2}-1} \otimes \omega^{a_{1}} \otimes \omega^{a_{0}+1}$ lying in the lower alcove) satisfy $F_{i} \in$ $\mathrm{JH}\left(\overline{R_{T}^{\theta_{i}}}\right)$, where

$$
\theta_{3}=[\omega]^{a_{2}-2} \otimes[\omega]^{a_{1}+1} \otimes[\omega]^{a_{0}+1}, \quad \theta_{4}=[\omega]^{a_{2}-1} \otimes[\omega]^{a_{1}-1} \otimes[\omega]^{a_{0}+2}
$$

and hence can not be modular by virtue of Theorem 5.5 Proposition 4.2 for Type $\left(\mathrm{T}_{2}\right)$. Similarly, the weights

$$
F\left(a_{2}-1, a_{1}+1, a_{0}\right), F\left(a_{1}+1, a_{0}, a_{2}-1-(p-1)\right)
$$

can not be modular as well. This completes the proof in Type $\left(\mathrm{T}_{2}\right)$ of the corollary.

Types $\left(\mathrm{T}_{0}\right)$ and $\left(\mathrm{T}_{1}\right)$ are deduced in a completely analogous fashion (using Proposition 4.2 specialized to the Types $\left(\mathrm{T}_{0}\right)$ and $\left(\mathrm{T}_{1}\right)$ respectively): we leave the computational details to the reader.

5.4.2. Niveau 2 types. We keep the assumption and the notation as at the beginning of Section 5.4.

Proposition 5.12. If $V=\bigotimes_{v^{\prime} \mid p} V_{v^{\prime}}$ is a modular weight for $\bar{r}$, then $F=V_{v} \circ \iota_{w}^{-1}$ is isomorphic to one of the weights in the following list

$$
F\left(a_{0}+(p-1), a_{1}, a_{2}-(p-1)\right), \quad F\left(a_{2}-1, a_{1}, a_{0}+1\right), \quad F\left(a_{2}-1, a_{0}, a_{1}+1-(p-1)\right)
$$

with moreover, according to the isomorphism class of $\bar{\rho}$, the additional weights:

$$
F\left(a_{1}+(p-1), a_{2}, a_{0}\right), \quad F\left(a_{0}-1+(p-1), a_{2}, a_{1}\right), \quad F\left(a_{2}, a_{0}, a_{1}-(p-1)\right)
$$

if $\bar{\rho}$ is of Type $\left(\mathrm{T}_{1}\right)$, and the further weight:

$$
F\left(a_{1}-1+(p-1), a_{2}, a_{0}+1\right)
$$

if $\bar{\rho}$ is of Type $\left(\mathrm{T}_{0}\right)$.

For the proof of Proposition 5.12 we record the following easy lemma:

Lemma 5.13. Let $F=F(x, y, z)$ be a restricted weight lying in the upper alcove and let $i \geq j>k$ be integers such that $i-k \leq p-1$.

Let $\theta \cong[\omega]^{i} \otimes\left[\omega_{2}\right]^{j+p k}$ via the isomorphism $T\left(k_{w}\right) \cong k_{w}^{\times} \times k_{2, w}^{\times}$.

The relation

$$
F \in \mathrm{JH}\left(\overline{R_{T}^{\theta}}\right)
$$

holds true if and only if

$$
(i, j, k) \in\{(y, x-(p-2), z-1),(x, p-1+z, y),(p-1+z, y, x-(p-1))\} .
$$


Proof. It is an elementary exercise using [EGH13], Lemma 5.2.4 and the exhaustive description of the Jordan-Hölder factors of the mod- $p$ reduction of the Deligne-Lusztig representation $\operatorname{Ind}_{P_{2}\left(\mathbf{F}_{p}\right)}^{\mathrm{GL}_{3}\left(\mathbf{F}_{p}\right)}[\omega]^{i} \otimes \Theta\left(\left[\omega_{2}\right]^{j+p k}\right)$ performed in the proof of [Her09], Proposition 7.4.

Proof of Proposition 5.12. We perform in detail the proof for the Type $\left(\mathrm{T}_{2}\right)$ : Types $\left(\mathrm{T}_{0}\right)$ and $\left(\mathrm{T}_{1}\right)$ are proved in the evident similar fashion and we leave the computations as an exercise to the scrupulous reader.

In all what follows we identify $\theta: T\left(k_{w}\right) \rightarrow E^{\times}$with a character of the form $[\omega]^{x} \otimes[\omega]_{2}^{y}$ for some appropriate $x, y \in \mathbf{Z}$, via the isomorphism $T\left(k_{w}\right) \cong k_{w}^{\times} \times k_{2, w}^{\times}$.

Let us consider the following weights lying in the lower alcove:

$$
F\left(a_{0}+(p-1), a_{2}-1, a_{1}+1\right), \quad F\left(a_{1}, a_{0}, a_{2}-(p-1)\right) .
$$

By the explicit description of the Jordan-Hölder factors for Niveau 2 types it is easy to check that

$$
F\left(a_{0}+(p-1), a_{2}-1, a_{1}+1\right) \in \mathrm{JH}\left(\overline{R_{T}^{\theta_{1}}}\right) ; \quad F\left(a_{1}, a_{0}, a_{2}-(p-1)\right) \in \mathrm{JH}\left(\overline{R_{T}^{\theta_{2}}}\right)
$$

with

$$
\theta_{1} \cong[\omega]^{a_{2}-1} \otimes[\omega]_{2}^{a_{1}+p\left(a_{0}+1\right)} ; \quad \theta_{2} \cong[\omega]^{a_{0}} \otimes[\omega]_{2}^{a_{2}-1+p\left(a_{1}+1\right)} .
$$

By the first part of Proposition 4.3 and Theorem 5.5 we conclude that the weights (26) can not be modular for $\bar{r}$.

By Lemma 5.13 we obtain the following relations on the remaining weights in the upper alcove:

$$
\begin{aligned}
& F\left(a_{2}-1, a_{0}+1, a_{1}+(p-1)\right) \in \mathrm{JH}\left(\overline{R_{T}^{\theta_{1}}}\right), \\
& F\left(a_{1}+(p-1), a_{2}-1, a_{0}+1\right) \in \mathrm{JH}\left(\overline{R_{T}^{\theta_{2}}}\right), \\
& F\left(a_{0}+p, a_{1}, a_{2}-p\right) \in \mathrm{JH}\left(\overline{R_{T}^{\theta_{3}}}\right), \\
& F\left(a_{1}+p, a_{2}-1, a_{0}\right) \in \mathrm{JH}\left(\overline{R_{T}^{\theta_{4}}}\right), \\
& F\left(a_{0}+(p-1), a_{1}+1, a_{2}-p\right) \in \mathrm{JH}\left(\overline{R_{T}^{\theta_{5}}}\right) .
\end{aligned}
$$

where

$$
\begin{aligned}
\theta_{1}=\theta_{3}=[\omega]^{a_{2}-1} \otimes\left[\omega_{2}\right]^{a_{1}+p\left(a_{0}+1\right)}, & \theta_{2}=[\omega]^{a_{0}+p} \otimes\left[\omega_{2}\right]^{a_{2}-1+p a_{1}}, \\
\theta_{4}=[\omega]^{a_{2}-1} \otimes\left[\omega_{2}\right]^{a_{1}+2+p\left(a_{0}-1\right)}, & \theta_{5}=[\omega]^{a_{0}} \otimes\left[\omega_{2}\right]^{a_{1}+1+p\left(a_{2}-1\right)} .
\end{aligned}
$$

In view of the first part of Proposition 4.3 and Theorem 5.5 the weights in (27) can not be modular for $\bar{r}$.

To conclude, we see from Lemma 5.13 that the conditions

$$
F\left(a_{1}+(p-1), a_{1}, a_{0}\right) \in \mathrm{JH}\left(\overline{R_{T}^{\theta_{1}}}\right), \quad F\left(a_{2}, a_{0}, a_{1}-(p-1)\right) \in \mathrm{JH}\left(\overline{R_{T}^{\theta_{2}}}\right) .
$$

imply

$$
\theta_{1}=[\omega]^{a_{2}} \otimes\left[\omega_{2}\right]^{a_{1}+1+p\left(a_{0}-1\right)}, \quad \theta_{2}=[\omega]^{a_{0}+(p-1)} \otimes\left[\omega_{2}\right]^{a_{2}+1+p\left(a_{1}-1\right)}
$$

and both types in the RHS are not allowed to produce modular weights for $\bar{r}$, by virtue of the second part of Proposition 4.3 and Theorem 5.5.

Together with Proposition 5.11, this ends the proof of Proposition 5.12 for Type $\left(\mathrm{T}_{2}\right)$. 
Remark 5.14. Note that Proposition 5.12 together with the identity (23) completes the proof of Theorem 5.9 in the non-split case.

The same argument as in Propositions 5.11 and 5.12 works for $\left.\bar{r}\right|_{G_{F_{w}}}$ semi-simple, by using only $\bar{\rho}^{\text {ss }}$-part of Propositions 4.2 and 4.3. We leave this routine computation to the reader, and this completes the proof of Theorem 5.9.

5.4.3. Niveau 3 types. The goal of this subsection is to check if we can further eliminate some of the weights appearing in Proposition 5.12 using niveau 3 types and the splitting lemma. In fact, all of the weights in Proposition 5.12 survives. We consider more in detail the shadow weight $F\left(a_{2}, a_{0}, a_{1}-(p-1)\right.$ ), appearing as a possible (local) modular weight for $\bar{r}$ when $\left.\bar{\rho} \stackrel{\text { def }}{=} \bar{r}\right|_{G_{F_{w}}}$ is of Type $\left(\mathrm{T}_{1}\right)$. One could check, in an evident similar fashion, that the other weights in Proposition 5.12 survive as well.

We fix a maximal torus $T / \mathcal{O}_{F_{w, 3}}$ such that $T\left(k_{w}\right) \cong k_{w, 3}^{\times}$; this lets us identify a character $\theta: T\left(k_{w}\right) \rightarrow E^{\times}$with $\left[\omega_{3}\right]^{a(\theta)}: k_{w, 3}^{\times} \rightarrow E^{\times}$for some $a(\theta) \in \mathbf{N}$, where $\omega_{3}$ is a Serre fundamental character of niveau 3.

In the following remark, we record a concrete statement we want to check in this subsection.

Remark 5.15. Assume that $\bar{\rho}$ is of Type $\left(\mathrm{T}_{1}\right)$ and let $\theta: T\left(k_{w}\right) \rightarrow E^{\times}$be a primitive character such that

$$
F\left(a_{2}, a_{0}, a_{1}-(p-1)\right) \in \mathrm{JH}\left(\overline{R_{T}^{\theta}}\right) .
$$

Let $\rho_{0}: G_{\mathbf{Q}_{p}} \rightarrow \mathrm{GL}_{3}(E)$ be a potentially crystalline p-adic Galois representation with HodgeTate weights $\{0,-1,-2\}$ having Galois type $\operatorname{rec}(\theta)$ and $\mathcal{M} \in \mathbf{F}$-BrMod ${ }_{\mathrm{dd}}^{2}$ be a rank-three Breuil module deduced from $\rho_{0}$ via base change $S_{\mathcal{O}_{E}} \rightarrow \overline{\mathrm{S}}_{\mathbf{F}}$ and Theorem 2.1.

Then the condition $\bar{\rho}_{0}=\mathrm{T}_{\mathrm{st}}^{*}(\mathcal{M}) \cong \bar{\rho}$ does not lead to a contradiction with Proposition 4.4 nor with Lemma 3.2.

In particular, the weight elimination technique as developed in this paper does not let one eliminate the weight $F\left(a_{2}, a_{0}, a_{1}-(p-1)\right)$. The remaining of this subsection is devoted to check Remark 5.15. To ease notations and computation we may, and do, assume that $a_{0}=0$ and write $a \stackrel{\text { def }}{=} a_{2}, b \stackrel{\text { def }}{=} a_{1}$ (the general case follows by twisting by $[\omega]^{a_{0}}$ ).

We first determine the primitive niveau 3 character $\theta: T\left(k_{w, 3}\right) \rightarrow E^{\times}$appearing in the statement of Remark 5.15.

Lemma 5.16. Let $\theta \cong\left[\omega_{3}\right]^{i+p j+p^{2} k}: T\left(k_{w, 3}\right) \rightarrow E^{\times}$be a primitive character. If $F \stackrel{\text { def }}{=}$ $F(a, 0, b-(p-1)) \in \mathrm{JH}\left(\overline{R_{T}^{\theta}}\right)$ then $R_{T}^{\theta}$ is determined by either one of the following:

(i) $i>j \geq k, i-k \leq p$ and $\theta \cong\left[\omega_{3}\right]^{(a+1)+p(b-1)}$;

(ii) $i<j \leq k, k-i \leq p$ and $\theta \cong\left[\omega_{3}\right]^{(b-1)+p(a+1)+p^{2}(p-1)}$.

Proof. Let us write $\theta=\left[\omega_{3}\right]^{i+p j+p^{2} k}$ with $p^{2}+p+1 \nmid i+p j+p^{2} k$.

We start from (i). As the triple $(a, b, 0)$ is strongly generic, we deduce from the decomposition (7.8) in [Her09] that $F \in\{F(2(p-1)+k, p-1+j, i), F(p-1+j, i-1, k+1), F(i-$ $1, k, j+1-(p-1))\}$. By [EGH13], Lemma 5.2.4 one deduces (i).

As for (ii), we have $\left(R_{T}^{\theta}\right)^{\vee} \cong R_{T}^{\theta^{-1}}$ (cf. for instance [Her09], proof of Proposition 6.23). Since $(-i)>(-j) \geq(-k),-i+k \leq p$ we can use again the decomposition (7.8) in [Her09] (and dualize back) to obtain $F \in\{F(i, j-(p-1), k-2(p-1)), F(k-1, i+1, j-(p-$ $1)), F(j+(p-2), k, i+1)\}$. The conclusion follows from a straightforward computation via [EGH13], Lemma 5.2.4. 
For each case of Lemma 5.16, we need to check that there is a possible lift in Proposition 4.4 which we can not eliminate by the splitting Lemma 3.2. More precisely, let $\mathcal{M} \in \mathbf{F}$-BrMod ${ }_{\mathrm{dd}}^{2}$ be a rank-three Breuil module with niveau three descent data and Galois type $\operatorname{rec}(\theta)$ with $\theta: T\left(k_{w, 3}\right) \rightarrow E^{\times}$as either case in Lemma 5.16. Assume that $\mathrm{T}_{\mathrm{st}}^{*}(\mathcal{M}) \cong \bar{\rho}$ and let $\mathcal{M}_{2,1}$ be the Breuil submodule of $\mathcal{M}$ corresponding (as in Section 4.1) to the extension $\omega^{a+1}-\omega^{b+1}$ appearing in $\bar{\rho}$. Recall that $\bar{\rho}$ is of Type $\left(\mathrm{T}_{1}\right)$. Then we need to check that the splitting Lemma does not apply to the extension $0 \rightarrow \mathcal{M}_{2} \rightarrow \mathcal{M}_{2,1} \rightarrow \mathcal{M}_{1} \rightarrow 0$

In the case (i) of Lemma 5.16, it is relatively easy to check. Since one can not apply the splitting Lemma 3.2 in the case $k_{2} \equiv p k_{1} \bmod e=p^{3}-1$, we show that there exists $\delta_{i}$, defined in Proposition 4.4, such that $r_{i}$ are sitting between 0 and $2\left(p^{2}+p+1\right)$ when $k_{2} \equiv p k_{1}$ mod $e$; this lets us check Remark 5.15 in the case (i) of Lemma 5.16.

Indeed, we let $x=p[(a+1)+p(b-1)]$ and take $\delta_{2}=(p-1)(b-1), \delta_{1}=0$, and $\delta_{0}=(p-1)[a+1+p(b-1)]$. Then one can readily check that $x=\left(\delta_{2}+a\right)+\left(\delta_{1}+b\right)+\delta_{0}$ and the corresponding $r_{i}$ are sitting between 0 and $2\left(p^{2}+p+1\right)$.

In the case (ii) of Lemma 5.16, we need a bit more computation. In this case, one can readily check that there are no $\delta_{i}$ such that $0 \leq r_{i} \leq 2\left(p^{2}+p+1\right)$ in Proposition 4.4 when $k_{2} \equiv p k_{1} \bmod e$. However, in the case $k_{2} \equiv x \bmod e$ and $k_{1} \equiv p x \bmod e$ for $x=a+1+p(p-1)+p^{2}(b-1)$ (and so case (i) in Proposition 4.4), there exist $\delta_{i}$ such that $0 \leq r_{i} \leq 2\left(p^{2}+p+1\right)$. If we take $\delta_{2}=(p-1)(p b-1), \delta_{1}=0$, and $\delta_{0}=(p-1) b$, then $x=\left(\delta_{2}+a\right)+\left(\delta_{1}+b\right)+\delta_{0}$ and the corresponding $r_{i}$ are

$$
\left\{\begin{array}{l}
r_{2}=p(a-b+1)+a+2 \\
r_{1}=p^{2}+p(b+1)+p-(a-b) \\
r_{0}=\left(p^{2}+p+1\right)+p(p-1)-p a-b
\end{array}\right.
$$

which are obviously between 0 and $2\left(p^{2}+p+1\right)$. But, in this case, we can not apply the splitting lemma 3.2 either.

Indeed, we let $s_{x}=r_{2}, s_{y}=r_{1}, k_{x}=k_{2}$, and $k_{y}=k_{1}$, and try to apply the Lemma 3.2 in this case. It is easy to check that $p\left(s_{y}-s_{x}\right)+\left[k_{y}-k_{x}\right]_{3}>0$ and $j_{0}=0$, which follows immediately from $\left[k_{y}-k_{x}\right]_{3}=p^{3}-p^{2}(b+2)+p(a+2)-a+b-2$ and $\left[p^{-1} k_{y}-k_{x}\right]_{3}=0$ respectively. But $2\left(j_{0}+e\right)+\left[p^{-1} k_{y}-k_{x}\right]_{3}=2 e>(p-1)\left[\left(p^{2}+p+1\right)+p(a+2)+b+1\right]=$ $(p-1)\left(s_{x}+s_{y}\right)$, i.e., it violates the inequality (5) in the Lemma 3.2.

Hence, we have checked the Remark 5.15.

Remark 5.17. The techniques developed in this paper do not pertain only to the threedimensional case. In ongoing work, we are considering the case of an n-dimensional, ordinary, and strongly generic Galois representation $\bar{\rho}: G_{\mathbf{Q}_{p}} \rightarrow \mathrm{GL}_{n}(\mathbf{F})$, obtaining partial results when $\ell \ell(\bar{\rho}) \leq 3$.

\section{ACKNOWLEDGEMENTS}

The authors express their deepest gratitude to Florian Herzig for his kind suggestion to work on this problem, his encouragements, his many remarks, and his constant interest. We sincerely thank Daniel Le for his many precious comments and suggestions.

The authors started working on this project when both were postdoctoral fellows in the University of Toronto. The authors would like to thank the university for the warm hospitality. Part of this paper was written when the second author was a postdoc in Max Planck Institute for Mathematics in Bonn, and he also thanks the institute for providing him with an excellent research environment. 


\section{REFERENCES}

[ADP02] Avner Ash, Darrin Doud, and David Pollack, Galois representations with conjectural connections to arithmetic cohomology, Duke Math. J. 112 (2002), no. 3, 521-579. MR 1896473 (2003g:11055)

[BDJ10] K. Buzzard, F. Diamond, and F. Jarvis, On Serre's conjecture for mod $\ell$ Galois representations over totally real fields, Duke Math. J. 155 (2010), no. 1, 105-161. MR 2730374 (2012k:11067)

$[\mathrm{BH}] \quad$ C. Breuil and F. Herzig, Ordinary representations and fundamental algebraic representations, Duke Math. J., to appear, preprint (2012).

[BLGG] Thomas Barnet-Lamb, Toby Gee, and David Geraghty, Serre weights for U(n), preprint (2014).

[BLGGT] Thomas Barnet-Lamb, Toby Gee, David Geraghty, and Richard Taylor, Potential automorphy and change of weight, Annals of Math, to appear.

[BM02] C. Breuil and A. Mézard, Multiplicités modulaires et représentations de $\mathrm{GL}_{2}\left(\mathbf{Z}_{p}\right)$ et de $\operatorname{Gal}\left(\overline{\mathbf{Q}}_{p} / \mathbf{Q}_{p}\right)$ en $l=p$, Duke Math. J. 115 (2002), no. 2, 205-310, With an appendix by Guy Henniart. MR 1944572 (2004i:11052)

[Bre] C. Breuil, Sur un problème de compatibilité locale-globale pour $\mathbf{G L}_{2}$, J. Reine Angew. Math. (to appear), avec une appendice par L- Dembelé.

[Bre99] Christophe Breuil, Une application de corps des normes, Compositio Math. 117 (1999), no. 2, 189-203. MR 1695849 (2000f:11157)

[Car] A. Caraiani, Monodromy and local-global compatibility for $\ell=p$, Algebra and Number Theory, to appear.

[CDT99] Brian Conrad, Fred Diamond, and Richard Taylor, Modularity of certain potentially barsotti-tate galois repre- sentations, J. Amer. Math. Soc. 12 (1999), 521-367.

[CHT08] Laurent Clozel, Michael Harris, and Richard Taylor, Automorphy for some l-adic lifts of automorphic mod l Galois representations, Publ. Math. Inst. Hautes Études Sci. (2008), no. 108, 1-181, With Appendix A, summarizing unpublished work of Russ Mann, and Appendix B by Marie-France Vignéras. MR 2470687 (2010j:11082)

[CL09] Xavier Caruso and Tong Liu, Quasi-semi-stable representations, Bull. Soc. Math. France 137 (2009), no. 2, 185-223. MR 2543474 (2011c:11086)

[EGH13] M. Emerton, T. Gee, and F. Herzig, Weight cycling and Serre-type conjectures for unitary groups, Duke Math. J. 162 (2013), no. 9, 1649-1722. MR 3079258

[EGHS] M. Emerton, T. Gee, F. Herzig, and D. Savitt, General serre weight conjectures, in preparation.

[FL82] Jean-Marc Fontaine and Guy Laffaille, Construction de représentations p-adiques, Ann. Sci. École Norm. Sup. (4) 15 (1982), no. 4, 547-608 (1983). MR 707328 (85c:14028)

[Fon90] Jean-Marc Fontaine, Représentations p-adiques des corps locaux. II, The Grothendieck Festschrift, Vol. II, Progr. Math., vol. 87, Birkhäuser Boston, Boston, MA, 1990, pp. 249-309. MR 1106901 (92i:11125)

[Fon94] _ Représentations $\ell$-adiqes potentiellement semistables, Astérisque 223 (1994), 321-347.

[Gee11] Toby Gee, Automorphic lifts of prescribed types, Math. Ann. 350 (2011), no. 1, 107-144. MR 2785764 (2012c:11118)

[GG12] Toby Gee and David Geraghty, Companion forms for unitary and symplectic groups, Duke Math. J. 161 (2012), no. 2, 247-303. MR 2876931

[GK] T. Gee and M. Kisin, The breuil-mézard conjecture for potentiallly barsotti-tate representations, Forum of Math, Pi, to appear.

[GLS] Toby Gee, Tong Liu, and David Savitt, The weight part of serre's conjecture for GL(2), preprint (2014).

[GLS14] The Buzzard-Diamond-Jarvis conjecture for unitary groups, J. Amer. Math. Soc. 27 (2014), no. 2, 389-435. MR 3164985

[Hen00] Guy Henniart, Une preuve simple des conjectures de Langlands pour GL $(n)$ sur un corps padique, Invent. Math. 139 (2000), no. 2, 439-455. MR 1738446 (2001e:11052)

[Her09] Florian Herzig, The weight in a Serre-type conjecture for tame $n$-dimensional Galois representations, Duke Math. J. 149 (2009), no. 1, 37-116. MR 2541127 (2010f:11083)

[HM] F. Herzig and S. Morra, On mod $p$ local-global compatibility for $\mathrm{GL}_{3}$ in the ordinary case, In preparation (2014).

[HT01] Michael Harris and Richard Taylor, The geometry and cohomology of some simple Shimura varieties, Annals of Mathematics Studies, vol. 151, Princeton University Press, Princeton, NJ, 2001, With an appendix by Vladimir G. Berkovich. MR 1876802 (2002m:11050)

[Le] Daniel Le, Aspects of the p-adic Langlands program for $\mathrm{GL}_{3}\left(\mathbf{Q}_{p}\right)$ in families, $\mathrm{PhD}$ thesis. 
[Liu08] T. Liu, On lattices in semi-stable representations: a proof of a conjecture of Breuil, Compos. Math. 144 (2008), no. 1, 61-88. MR 2388556 (2009c:14087)

[Mor99] Lawrence Morris, Level zero G-types, Compositio Math. 118 (1999), no. 2, 135-157. MR 1713308 (2000g:22029)

[PR94] Bernadette Perrin-Riou, Représentations p-adiques ordinaires, Astérisque (1994), no. 223, 185220, With an appendix by Luc Illusie, Périodes $p$-adiques (Bures-sur-Yvette, 1988). MR 1293973 (96h:11063)

[Ser87] J-P. Serre, Sur les représentations modulaires de degré 2 de Gal( $\overline{\mathbf{Q}} / \mathbf{Q})$, Duke Math. J. 54 (1987), no. 1, 179-230. MR 885783 (88g:11022)

The University of Toronto, 40 St. George Street, Toronto, Canada M5S 2E4

\& Université de Montpellier 2, place Eugène Bataillon, 34095 Montpellier, France

E-mail address: smorra@math.toronto.edu

Max Planck Institute for Mathematics, Vivatsgasse 7, Bonn 53111, Germany

E-mail address: cpark@mpim-bonn.mpg.de 